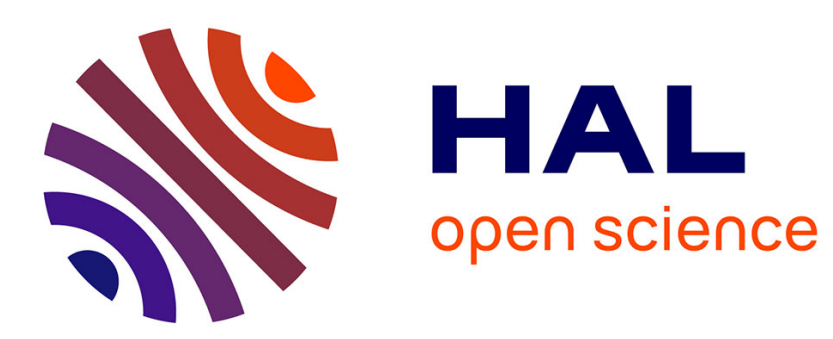

\title{
Topology optimization of particle-matrix composites for optimal fracture resistance taking into account interfacial damage
}

Daicong Da, Julien Yvonnet, Liang Xia, Guangyao Li

\section{- To cite this version:}

Daicong Da, Julien Yvonnet, Liang Xia, Guangyao Li. Topology optimization of particle-matrix composites for optimal fracture resistance taking into account interfacial damage. International Journal for Numerical Methods in Engineering, 2018, 115 (5), pp.604-626. 10.1002/nme.5818 . hal-01780793

\section{HAL Id: hal-01780793 \\ https://hal.science/hal-01780793}

Submitted on 16 May 2018

HAL is a multi-disciplinary open access archive for the deposit and dissemination of scientific research documents, whether they are published or not. The documents may come from teaching and research institutions in France or abroad, or from public or private research centers.
L'archive ouverte pluridisciplinaire HAL, est destinée au dépôt et à la diffusion de documents scientifiques de niveau recherche, publiés ou non, émanant des établissements d'enseignement et de recherche français ou étrangers, des laboratoires publics ou privés. 


\title{
Topology optimization of particle-matrix composites for optimal fracture resistance taking into account interfacial damage
}

\author{
Daicong $\mathrm{Da}^{1,2}$, Julien Yvonnet ${ }^{2 *}$, Liang $\mathrm{Xia}^{3}$, Guangyao $\mathrm{Li}^{1 *}$ \\ 1 State Key Laboratory of Advanced Design and Manufacturing for Vehicle Body, Hunan University, Changsha, China \\ ${ }^{2}$ Université Paris-Est, Laboratoire Modélisation et Simulation Multi Echelle MSME, UMR CNRS 8208, \\ 5 bd Descartes, 77454 Marne-la-Vallée, France \\ ${ }^{3}$ State Key Laboratory of Digital Manufacturing Equipment and Technology, Huazhong University of Science and \\ Technology, Wuhan, China
}

\begin{abstract}
SUMMARY
This paper presents a topology optimization framework for optimizing the fracture resistance of two-phase composites considering interfacial damage interacting with crack propagation through a redistribution of the inclusions phase. A phase field method for fracture capable of describing interactions between bulk brittle fracture and interfacial damage is adopted within a diffuse approximation of discontinuities. This formulation avoids the burden of remeshing problem during crack propagation and is well adapted to topology optimization purpose. Efficient design sensitivity analysis is performed by using the adjoint method, and the optimization problem is solved by an extended bi-directional evolutionary structural optimization (BESO) method. The sensitivity formulation accounts for the whole fracturing process involving cracks nucleation, propagation and interaction, either from the interfaces and then through the solid phases, or the opposite. The spatial distribution of material phases are optimally designed using the extended BESO method to improve the fractural resistance. We demonstrate through several examples that the fracture resistance of the composite can be significantly increased at constant volume fraction of inclusions by the topology optimization process.
\end{abstract}

Received ...

KEY WORDS: Cracks; Phase field method; Interfacial damage; Topology optimization; BESO

\section{INTRODUCTION}

Mechanical and physical properties of complex heterogeneous materials are determined on one hand by the composition of their constituents, but can on the other hand be drastically modified, at a constant volume fraction of heterogeneities, by their geometrical shape and by the presence of interfaces. For example, it is well known that in the context of linear properties, modifying

\footnotetext{
${ }^{*}$ Correspondence to: J. Yvonnet, Université Pris-Est, Laboratoire Modélisation et Simulation Multi Echelle MSME, CNRS UMR 8208, 5 bd Descartes, 77454 Marne-la-Vallée, France. E-mail: julien.yvonnet@univ-paris-est.fr; G.Y. Li, State Key Laboratory of Advanced Design and Manufacturing for Vehicle Body, Hunan University, Changsha, China. E-mail: gyli@hnu.edu.cn
} 


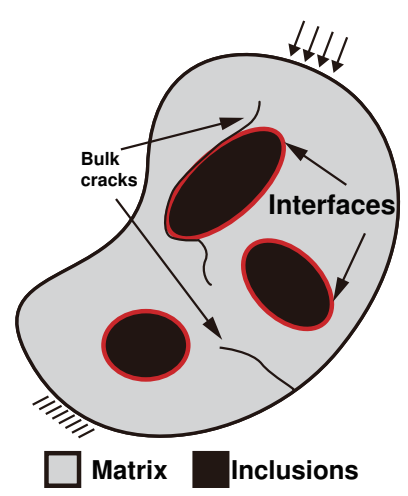

(a)

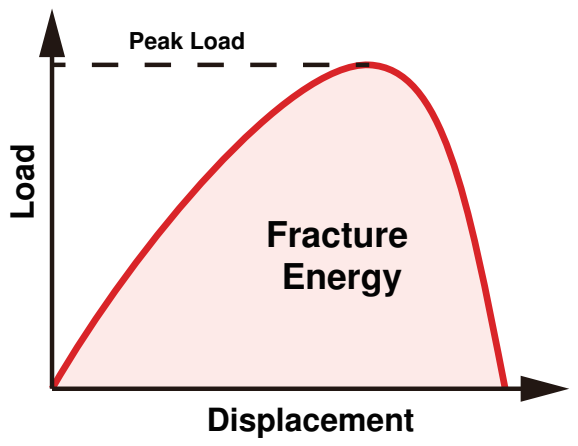

(b)

Figure 1. (a) structure containing both bulk cracks and interface cracks, possibly occurring at the interfaces, (b) mechanical response of the damageable structure.

the geometrical topology of phases at a constant volume fraction can induce a change in the maximum values of effective moduli, but also induces anisotropy. In that context, topological optimization of microstructures can help designing materials with higher effective properties while maintaining the volume fraction of constituents, or to obtain new properties which are not naturally available (metamaterials). Recently, the development of 3D printing techniques and other additive manufacturing processes (see e.g. [1]) have made possible to manufacture directly the designed materials from a numerical file, opening routes for totally new designs. Topological optimization of microstructures has been extensively studied in the linear context (see e.g. a review in [2] and the reference therein), and more recently in the nonlinear context (see e.g. [3]). One major challenge is to use topological optimization to improve the fracture resistance of heterogeneous materials, taking into account the heterogeneities and their interfaces in the material.

Optimization design of composite materials accounting for fracture resistance remains relatively unexplored so far, mainly due to the lack of robust numerical methods for fracture propagation in presence of complex heterogeneous media and interfaces, until recently. In addition, these numerical simulation models should be formulated in a context compatible with topological optimization (e.g. finite elements). In [4], Gu et al. used a modified greedy optimization algorithm for composites made up of soft and stiff building blocks to improve material toughness. San and Waisman [5] explored the optimal location of carbon black particle to maximize the rupture resistance of polymer composites by a genetic algorithm. In a recent work by the authors [6], topology optimization for maximizing the fracture resistance of quasi-brittle composites has been introduced by combining the phase field method and a gradient-based (BESO) algorithm. However, in the mentioned work, the crack propagation resistance was only evaluated on the basis of phase distribution. In most heterogeneous quasi-brittle materials (e.g. ceramic matrix composites, cementitious materials), the interfacial damage plays a central role in the nucleation and propagation of microcracks $[7,8,9,10]$. The main objective of the present work is to extend the framework developed in [6] for defining through topological optimization the optimal phase distribution in a quasi-brittle composite with respect to fracture resistance, taking into account crack nucleation both in the matrix and in the interfaces, as described in Fig. 1. To our best knowledge, such study is investigated here for the first time. 
Simulating interfacial damage and its interaction with matrix crack for complex heterogeneous materials is a highly challenging issue for meshing algorithms. Many numerical methods such as eXtended Finite Element Method (XFEM) [11, 12], Thick Level-set method (TLS) [13, 14], and Phase Field Method (PFM) [15, 16, 17], among the most recent popular techniques, have been introduced to investigate this topic.

Based on the pioneer works of Marigo and Francfort [15], the phase field method makes use of a variational principle framework for brittle fracture $[18,19,20]$ and of a regularized description of the discontinuities related to the crack front [21,22]. The method has been adapted to a convenient algorithmic setting by Miehe et al. in [23]. The main advantages of the method are: (a) the crack paths are mesh-independent; (b) initiation and propagation of multiple, complex cracks patterns can be easily handled; (c) the method is convergent with respect to the mesh size and not sensitive to the mesh regularity; (d) it is stable due to its inherent gradient-based formulation. In the present work, we use the extension of the phase field to interfacial damage as proposed in [24] to take into account both bulk brittle fracture and interfacial damage.

During the past decades, the research in topology optimization witnessed tremendous development since the seminal paper by Bensoe and Kikuchi [25]. So far, various topology optimization methods have been proposed, e.g., density-based method [26, 27, 28], evolutionary procedures [29, 30], level-set methods [31, 32, 33]. The extended bi-directional evolutionary structural optimization (BESO) method developed for the design of elastoplastic structure in [3, 34] is adopted in this work to carry out the topology optimization. A computationally efficient adjoint sensitivity formulation accounting for the complete fracturing process is derived, involving cracks nucleation, propagation and merging of microcracks until complete failure of the specimen. By using discrete design variables, the ESO-type methods omit naturally the definition of additional pseudo-relationships between fictitious materials and fracture toughness, resulting in a clear physical interpretation and algorithmic advantages [6, 35, 36, 37]. While there do exist numerous researches on topology optimization including material interface behavior (see e.g. [38, 39, 40, 41]), and with the enforcement of stress constraints [42, 43, 44, 45, 46, 47, 48], local buckling constraint [49] or damage constraints [50, 51, 52, 53, 54, 55], topology optimization for maximizing the fracture resistance, taking into account interactions between interfacial damage and bulk brittle fracture for complete fracturing process is to our best knowledge explored for the first time in the present work.

The layout of the paper is organized as follows. Section 2 gives the detailed phase field framework incorporating bulk fracture and cohesive interface. Numerical details and FEM discretization details are presented. In Section 3, we formulate the topology optimization model, containing detailed sensitivity derivation as well as the updating scheme with a damping on the sensitivity numbers. In Section 4, several numerical benchmark tests are presented to demonstrate the potential of the proposed method. Finally, conclusions are drawn in Section 5.

\section{PHASE FIELD MODELING OF BULK CRACK AND COHESIVE INTERFACES}

In this section we described the numerical method which is used by the topological optimization algorithm to obtain the fracture energy of the sample. The technique is based on the phase field 


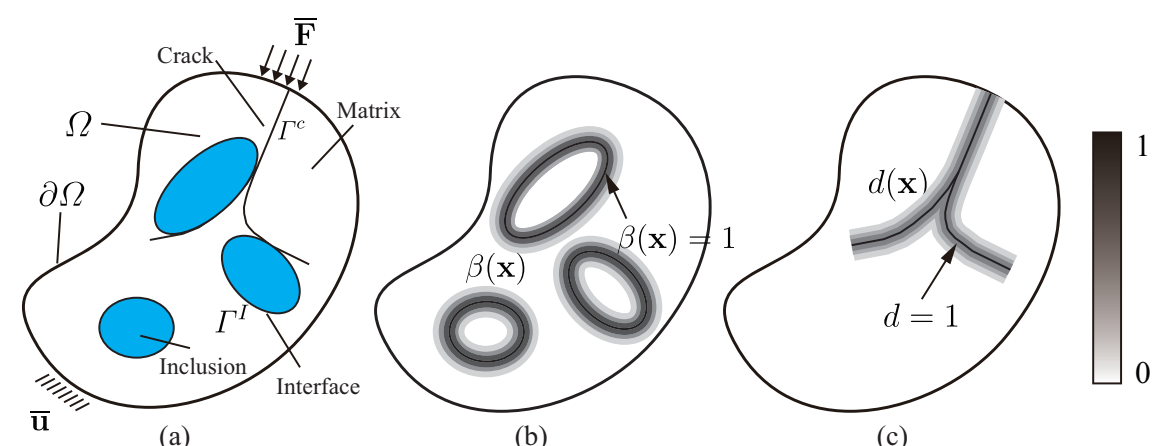

Figure 2. Illustration of the regularized representation of cracks and interfaces: (a) a solid containing interfaces and cracks; (b) regularized representation of the interfaces; (c) regularized representation of the cracks.

model for fracture extended to interfacial damage by Nguyen et al. [24], and allows simulating the initiation and propagation of a multiple cracks network in heterogeneous microstructures. The main concepts and details are recalled in the following.

\subsection{Regularized representation of discontinuous field}

Let $\Omega \in \mathbb{R}^{d}$ be an open domain describing a solid with external boundary $\partial \Omega$. The solid contains internal material interfaces between different phases, collectively denote by $\Gamma^{I}$. During the loading, cracks may propagate within the solid and can pass through the material interfaces as depicted in Fig. 2 (a). The crack surfaces are denoted by $\Gamma^{c}$. In this work, we adopt smeared representations of both cracks and material interfaces: , the cracks are approximated by an evolving phase field $d(\mathbf{x}, t)$. Interfaces between different material phases are described by a fixed scalar phase field $\beta(\mathbf{x})$. The material interfaces do not evolve during the loading. The regularized parameters describing the actual widths of the smeared cracks and material interfaces are respectively denoted by $\ell_{d}$ and $\ell_{\beta}$. In the following, the same regularization length $\ell=\ell_{\beta}=\ell_{d}$ is adopted for cracks and material interfaces for the sake of simplicity.

Given a non-evolving sharp crack defined on a surface $\Gamma^{c}$, a regularized (smeared) representation of the corresponding damage $d(\mathbf{x})$ (see Fig. 2 (c)) can be obtained by solving the following equations (see [16] for more details):

$$
\left\{\begin{array}{l}
d(\mathbf{x})-\ell^{2} \nabla^{2} d(\mathbf{x})=0, \text { in } \Omega \\
d(\mathbf{x})=1, \text { on } \Gamma^{c} \\
\nabla d(\mathbf{x}, t) \cdot \mathbf{n}=0, \text { on } \partial \Omega
\end{array}\right.
$$

where $\nabla^{2}($.$) is the Laplacian, \ell$ is the regularization parameter, and $\mathbf{n}$ is the unit outward normal to the external boundary $\partial \Omega$. It can be shown that Eq. (1) is the Euler-Lagrange equation corresponding to the variational problem:

$$
d(\mathbf{x}, t)=\operatorname{Arg}\left\{\inf _{d \in S_{d}} \Gamma^{d}(d)\right\}, \quad \Gamma^{d}(d)=\int_{\Omega} \gamma_{d}(d) \mathrm{d} \Omega,
$$


where $S_{d}=\{d \mid d(\mathbf{x})=1, \forall \mathbf{x} \in \Gamma\}$, and $\Gamma^{d}(d)$ represents the total crack length per unit area in 2D and total crack area of per unit volume in $3 \mathrm{D}$. In (2), $\Gamma_{d}(d)$ is defined by

$$
\gamma_{d}(d(\mathbf{x}))=\frac{1}{2 \ell} d(\mathbf{x})^{2}+\frac{\ell}{2} \nabla d(\mathbf{x}) \cdot \nabla d(\mathbf{x}) .
$$

When $\ell \rightarrow 0$, the above variation principle leads to the corresponding one with a sharp crack description in the $\Gamma$ - convergence sense. The details of definition (3) can be found e.g. in [23].

In Nguyen et al. [24], an interface phase field has been introduced to describe in the same manner the discontinuities related to the damage of interfaces and obtained by solving the problem:

$$
\left\{\begin{array}{l}
\beta(\mathbf{x})-\ell^{2} \nabla^{2} \beta(\mathbf{x})=0, \text { in } \Omega \\
\beta(\mathbf{x})=1, \text { on } \Gamma^{I} \\
\nabla \beta(\mathbf{x}) \cdot \mathbf{n}=0, \text { on } \partial \Omega .
\end{array}\right.
$$

Eq. (4) corresponds to the Euler-Lagrange equation associated with the variational problem

$$
\beta(\mathbf{x}, t)=\operatorname{Arg}\left\{\inf _{\beta \in S_{\beta}} \Gamma^{\beta}(\beta)\right\}, \quad \Gamma^{\beta}(\beta)=\int_{\Omega} \gamma_{\beta}(\beta) \mathrm{d} \Omega
$$

where $S_{\beta}=\left\{\beta \mid \beta(\mathbf{x})=1, \forall \mathbf{x} \in \Gamma^{I}\right\}, \Gamma^{\beta}$ represents the total interface length, and $\gamma_{\beta}$ is defined by

$$
\gamma_{\beta}(\beta)=\frac{1}{2 l} \beta(\mathbf{x})^{2}+\frac{\ell}{2} \nabla \beta(\mathbf{x}) \cdot \nabla \beta(\mathbf{x}) .
$$

For $\ell \rightarrow 0$ the above variation principle leads to a sharp interface description. This function will be used as an indicator to particularize the damage model to the interfaces or to the bulk in the formulation described in following sections.

In addition, it is necessary to introduce an approximation for the displacement jump at the interfaces, to associate a damage model specific to the interfaces and different from the bulk. For this purpose, the following approximation has been proposed [24] using Taylor expansion of the displacement field around a point $\mathbf{x}$ located on the interface:

$$
\llbracket \mathbf{u}(\mathbf{x}) \rrbracket \simeq \mathbf{w}(\mathbf{x})=\mathbf{u}\left(\mathbf{x}+\frac{h}{2} \mathbf{n}^{I}\right)-\mathbf{u}\left(\mathbf{x}-\frac{h}{2} \mathbf{n}^{I}\right)=h \nabla(\mathbf{u}(\mathbf{x})) \mathbf{n}^{\mathbf{I}},
$$

where $\mathbf{w}(\mathbf{x})$ denotes the smoothed displacement jump approximation and $\mathbf{n}^{I}$ is an approximation of the normal to the interface $\Gamma^{I}$ at a point $\mathrm{x}$. Several techniques are possible to define this normal. For example, in [24], a level-set technique has been proposed (see more detailed in the mentioned paper). In [56], another definition using the possible modification of the interface by the damage related to bulk cracks was introduced.

\subsection{Energy functional}

The following total energy functional formulated is introduced for the solid body related to both cracks and interfaces:

$$
E=\int_{\Omega} W_{u}^{e}\left(\varepsilon^{e}(\mathbf{u}, \beta), d\right) d \Omega+\int_{\Omega}[1-\beta(\mathbf{x})] g_{c} \gamma_{d}(d) d \Omega+\int_{\Omega} \psi^{I}(\mathbf{w}) \gamma_{\beta}(\beta) d \Omega,
$$


where $g_{c}$ is the toughness and $\psi^{I}$ is a strain density function depending on the displacement jump across the interface $\Gamma^{I}$. Above, $\varepsilon^{e}$ is the bulk part of the infinitesimal strain tensor $\varepsilon$ which satisfies the following relationship:

$$
\varepsilon=\varepsilon^{e}+\bar{\varepsilon}
$$

where $\bar{\varepsilon}$ is the strain part induced by the smoothed jump at the interfaces such that $\bar{\varepsilon} \rightarrow 0$ away from the interfaces (see [56]), in which case we recover the energy functional for a cracked body without considering the interface behavior:

$$
E=\int_{\Omega} W_{u}^{e}\left(\varepsilon^{e}(\mathbf{u}), d\right) d \Omega+\int_{\Omega} g_{c} \gamma_{d}(d) d \Omega
$$

From Eq. (8), the free energy $W$ can be identified as

$$
W=W_{u}^{e}\left(\varepsilon^{e}(\mathbf{u}, \beta), d\right)+[1-\beta(\mathbf{x})] g_{c} \gamma_{d}(d)+\psi^{I}(\mathbf{w}, \boldsymbol{\alpha}) \gamma_{\beta}(\beta)
$$

Following [24], the elastic energy $W_{u}^{e}$ is defined as

$$
W_{u}^{e}=\psi_{e}^{+}\left(\varepsilon^{e}\right)[g(d)+k]+\psi_{e}^{-}\left(\varepsilon^{e}\right)
$$

where

$$
\varepsilon^{e}=\varepsilon^{e+}+\varepsilon^{e-}
$$

and

$$
\psi_{e}^{ \pm}(\varepsilon)=\lambda\left\langle\operatorname{tr}\left[\varepsilon^{e}\right]\right\rangle_{ \pm}^{2} / 2+\mu \operatorname{tr}\left[\varepsilon^{e \pm}\right]^{2}
$$

and

$$
\varepsilon^{e \pm}=\sum_{i=1}^{D}\left\langle\varepsilon^{i}\right\rangle_{ \pm} \mathbf{n}^{i} \otimes \mathbf{n}^{i} .
$$

The decomposition (11) ensures that damage is only induced by traction, which removes the complications related to auto-contact in the crack. In (14)-(15), $\langle x\rangle_{ \pm}=(x \pm|x|) / 2 . \varepsilon^{i}$ and $\mathbf{n}^{i}$ are respectively the eigenvalues and eigenvectors of $\varepsilon^{e} . \lambda$ and $\mu$ are initial Lamé coefficients and $k$ is a very small value to maintain the well-posedness of the system. The degradation function $g(d)$ is assumed to have a simple form $g(d)=(1-d)^{2}$. A reduced Clausius-Duhem inequality form related to the evolution of the damage parameter $d$ can be written as

$$
\mathcal{A} \dot{d} \geq 0, \quad \mathcal{A}=-\frac{\partial W}{\partial d} .
$$

A threshold function $F(\mathcal{A})$ is assumed such that no damage occurs in the form:

$$
F(\mathcal{A})=\mathcal{A} \leq 0
$$

The principle of maximum dissipation requires that dissipation $\mathcal{A} \dot{d}$ must be maximum under the constraint, i.e. $\dot{d}>0, F=0$. Therefore, it yields

$$
F=-\frac{\partial W}{\partial d}=-\frac{\partial W_{u}^{e}}{\partial d}-(1-\beta) g_{c} \delta \gamma(d)=0
$$


with the functional derivative [16]

$$
\delta \gamma(d)=\frac{d}{\ell}-\ell \Delta d
$$

It follows that when $\dot{d}>0$ then

$$
-2(1-d) \psi_{e}^{+}+(1-\beta) g_{c} \delta \gamma(d)=0
$$

To handle loading and unloading, the strain history function adopted in [16, 24] is employed here:

$$
\mathcal{H}(\mathbf{x}, t)=\max _{\tau \in[0, t]}\left\{\psi_{e}^{+}(\mathbf{x}, t)\right\}
$$

and (20) is substituted by

$$
-2(1-d) \mathcal{H}+(1-\beta) g_{c} \delta \gamma(d)=0
$$

\subsection{Displacement and phase field problems}

2.3.1. Weak form of the phase field problem Using (19), the evaluation of the crack field $d(\mathbf{x}, \mathbf{t})$ can be determined by solving the following phase field problem

$$
\left\{\begin{array}{l}
2(1-d) \mathcal{H}-(1-\beta) \frac{g_{c}}{\ell}\left(d-\ell^{2} \nabla^{2} d\right)=0, \text { in } \Omega \\
d(\mathbf{x})=1, \text { on } \Gamma^{c} \\
\nabla d(\mathbf{x}) \cdot \mathbf{n}=0, \text { on } \partial \Omega .
\end{array}\right.
$$

The associated weak form is obtained as (see [24]):

$$
\int_{\Omega}\left\{\left(2 \mathcal{H}+[1-\beta] \frac{g_{c}}{\ell}\right) d \delta d+[1-\beta] g_{c} \ell \nabla d \cdot \nabla(\delta d)\right\} d \Omega=\int_{\Omega} 2 \mathcal{H} \delta d d \Omega
$$

2.3.2. Weak form of the displacement problem Using the variational principle for minimizing the total energy $E$ with respect to the displacement $\mathbf{u}$, the weak form associated with the displacement problem can be formulated as

$$
\int_{\Omega} \frac{\partial W_{u}^{e}}{\partial \boldsymbol{\varepsilon}^{e}}: \boldsymbol{\varepsilon}^{e}(\delta \mathbf{u}) d \Omega+\int_{\Omega} \frac{\partial \psi^{I}(\mathbf{w})}{\delta \mathbf{w}} \cdot \partial \mathbf{w} \gamma_{\beta}(\beta) d \Omega=\int_{\Omega} \mathbf{f} \cdot \delta \mathbf{u} d \Omega+\int_{\partial \Omega_{F}} \overline{\mathbf{F}} \cdot \delta \mathbf{u} d \Gamma=\delta W^{e x t} .
$$

In the absence of body forces, Eq.(25) can be re-written as

$$
\int_{\Omega} \boldsymbol{\sigma}^{e}: \boldsymbol{\varepsilon}^{e}(\delta \mathbf{u}) d \Omega+\mathbf{t}(\mathbf{w}) \cdot \delta \mathbf{w} \gamma_{\beta}(\beta) d \Omega-\int_{\Omega} \boldsymbol{\sigma}^{e}: \nabla^{s} \delta \mathbf{u} d \Omega=0
$$

where $\boldsymbol{\sigma}^{e}=\frac{\partial \mathbf{w}_{e}}{\partial \boldsymbol{\varepsilon}^{e}}$ is the Cauchy stress, and $\mathbf{t}(\mathbf{w})$ is the traction vector acting on the interface $\Gamma^{I}$ oriented by $\mathbf{n}^{I}$ and $\delta \mathbf{w}=h \nabla(\delta \mathbf{u}) \mathbf{n}^{I}$. Using $\boldsymbol{\sigma}^{e} \mathbf{n}=\mathbf{t}$, the above equation can be further re-written as

$$
\int_{\Omega} \boldsymbol{\sigma}^{e}:\left\{\boldsymbol{\varepsilon}^{e}(\delta \mathbf{u})+\mathbf{n} \otimes \delta \mathbf{w} \gamma_{\beta}(\beta)-\nabla^{s} \delta \mathbf{u}\right\} d \Omega
$$




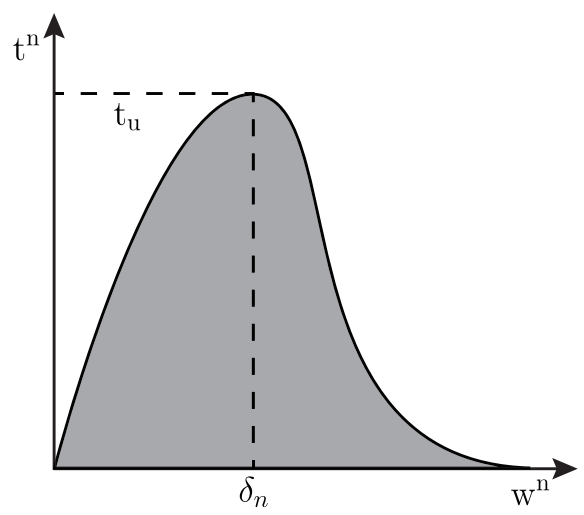

Figure 3. Illustration of the cohesive model for the interfaces.

which is satisfied for an admissible strain field:

$$
\varepsilon^{e}=\nabla^{s} \mathbf{u}-\mathbf{n} \otimes^{S} \mathbf{w} \gamma_{\beta}
$$

where $\left(\nabla^{s} \mathbf{u}\right)_{i j}=\left(u_{i, j}+u_{j, i}\right) / 2$ and $\left(\mathbf{n} \otimes{ }^{S} \mathbf{w}\right)_{i j}=\left(n_{i} \mathbf{w}_{j}+\mathbf{w}_{i} n_{j}\right)$. From (13), $\bar{\varepsilon}$ can be identified as $\bar{\varepsilon}=\mathbf{n} \otimes{ }^{S} \mathbf{w} \gamma_{\beta}$.

With the above description of strain energy function, the Cauchy stress now reads:

$$
\boldsymbol{\sigma}^{e}=\frac{\partial \psi_{e}^{+}}{\partial \boldsymbol{\varepsilon}^{e}}[g(d)+k]+\frac{\partial \psi_{e}^{-}}{\partial \boldsymbol{\varepsilon}^{e}}=\left[(1-d)^{2}+k\right]\left\{\lambda\left\langle\operatorname{tr} \varepsilon^{e}\right\rangle_{+} \mathbf{1}+2 \mu \varepsilon^{e+}\right\}+\lambda\left\langle\operatorname{tr} \varepsilon^{e}\right\rangle_{-} \mathbf{1}+2 \mu \varepsilon^{e-} .
$$

2.3.3. Model for interface damage The general form of the traction vector $\mathbf{t}(\mathbf{w})$ in Eq. (26) is given by

$$
\mathbf{t}(\mathbf{w})=\left[t^{n}, t^{t}\right]^{T}
$$

where $t^{n}$ and $t^{t}$ denote respectively normal and tangential parts of the traction vector $\mathbf{t}$ across the interface oriented by its normal $\mathbf{n}^{I}$. In the presented work, a simplified nonlinear elastic cohesive model is used. We have shown that the framework proposed in [24] allows avoiding the use of internal variables related to the interface damage to handle loading and unloading by exploiting the damage phase field itself as the history variable. In addition, by only taking into account the normal traction, i.e. $\mathbf{t}(\mathbf{w}) \cdot \mathbf{n}^{I}=t^{n}$, the cohesive law can be written as

$$
t^{n}=g_{c}^{I}\left(\frac{w^{n}}{\delta^{n}}\right) \exp \left(-\frac{w^{n}}{\delta^{n}}\right)
$$

where $w^{n}=\mathbf{w} \cdot \mathbf{n}^{I}$. For this model, we obtain $\mathbf{K}_{I}=\frac{\partial \mathbf{t}(\mathbf{w})}{\partial \mathbf{w}}$. The relationship between $\delta^{n}$, the toughness $g_{c}^{I}$ and th fracture strength $t_{u}$ is given by $\delta^{n}=g_{c}^{I} /\left(t_{u} e\right)$, with $e=\exp (1)$ (see Fig. 3).

Details about the linearization of the displacement problem and about FEM discretizations and numerical implementation are provided in Appendix 6. 


\section{TOPOLOGY OPTIMIZATION METHOD}

In this section, we present the topological optimization method based on the bi-directional evolutionary structural optimization (BESO) [30]. In the proposed procedure, the geometry of inclusions is optimized so as to maximize the fracture resistance of the sample. This procedure involves evaluating the sensitivity of the whole fracturing process (initiation of multiple cracks, propagation and complete failure of the sample) with respect to changes in the geometry.

\subsection{Model definitions}

The considered domain $\Omega$ is discretized into $N_{i}$ finite elements and each element $i$ is assigned with a topology design variable $\rho_{i}$. Within the framework of the bi-directional evolutionary structural optimization (BESO) method, the discrete design variables are defined as

$$
\rho_{i}=0 \text { or } 1, \quad i=1,2, \ldots, N_{\mathrm{i}}
$$

Following the multiple material interpolation model in $[57,58]$ we have

$$
E_{i}=\rho_{i} E_{\mathrm{inc}}+\left(1-\rho_{i}\right) E_{\mathrm{mat}}
$$

where $E_{\text {inc }}$ and $E_{\text {mat }}$ are the Young's moduli of the inclusion and matrix phases, respectively. The Poisson's ratios of the two material phases are assumed identical. The design variables can thus be interpreted as an indicator for the generalized material density. From (33), the density value of zero corresponds to the matrix phase, whereas one corresponds to the inclusion phase.

In nonlinear topology optimization, it is conventional to adopt displacement-controlled loading due to stability considerations [59, 60,61, 62]. For a prescribed displacement load, the objective of structural fracture resistance maximization is equivalent to maximization of the mechanical work during the fracturing process. In practice, the total mechanical work $J$ is calculated by using numerical integration, i.e.

$$
J \approx \frac{1}{2} \sum_{n=1}^{n_{\text {load }}}\left(\mathbf{f}_{\text {ext }}^{(n)}+\mathbf{f}_{\text {ext }}^{(n-1)}\right)^{T} \Delta \mathbf{u}^{(n)},
$$

and will be used as our definition to fracture resistance in the following. Above, $n_{\text {load }}$ is the total number of displacement increments, $\Delta \mathbf{u}^{(n)}$ is the $n$-th nodal displacement components and $\mathbf{f}_{\text {ext }}^{n}$ is the external nodal force at the $n$-th load increment.

During the optimization design, the material volume fractions of matrix and of inclusion phases are prescribed. Then the topology optimization problem subjected to balance equation and inclusion volume constraint can be formulated as

$$
\begin{aligned}
\max _{\boldsymbol{\rho}}: & J(\boldsymbol{\rho}, \mathbf{u}, \mathbf{d}, \boldsymbol{\beta}) \\
\text { subjected to }: & \mathcal{R}=0 \\
: & V(\boldsymbol{\rho})=\sum \rho_{i} v_{i}=V_{\text {req }} \\
: & \rho_{i}=0 \text { or } 1, i=1, \ldots, N_{\mathrm{i}}
\end{aligned}
$$


In the above, $v_{i}$ is the volume of $i$-th element, $V(\boldsymbol{\rho})$ and $V_{\text {req }}$ are the total and required material volumes, respectively and $\mathcal{R}$ denotes the nodal residual force:

$$
\mathcal{R}=\mathbf{f}_{\text {ext }}-\mathbf{f}_{\text {int }} .
$$

In (36), $\mathbf{f}_{\text {int }}$ is defined in each element as the internal force vector given in terms of the associated topology design variable $\rho_{i}$ and the Cauchy stress as:

$$
\mathbf{f}_{\mathrm{int}}=\sum_{i=1}^{N_{i}} \rho_{i} \int_{\Omega_{i}} \mathbf{B}^{T} \boldsymbol{\sigma}^{e} d \Omega_{i} .
$$

It should be mentioned that in this framework the discrete topology design variables are adopted to indicate the existence of the associated material phase (matrix/inclusion) of the element $i$. Then there is no need to define the physical meaning of the intermediate densities as is the case in the continuous density-based models (see e.g.,[26]) resulting in a clear physical interpretation.

\subsection{Sensitivity analysis}

In order to perform the topology optimization, the sensitivity of the objective function $J$ with respect to topology design variables $\rho$ must be computed. The adjoint method is employed to derive the sensitivity analysis. Firstly, two Lagrangian multipliers $\boldsymbol{\mu}^{(n)}, \boldsymbol{\lambda}^{(n)}$ of the same dimension as the vector of unknowns $\mathbf{u}$ are introduced to enforce zero residual $\mathcal{R}$ at time $t_{n-1}$ and $t_{n}$ for each term of the total mechanical work (34). Then the objective function $J$ can be rewritten in the following form without modifying the original objective value as

$$
\widehat{J}=\frac{1}{2} \sum_{n=1}^{n_{\text {load }}}\left\{\left(\mathbf{f}_{\text {ext }}^{(n)}+\mathbf{f}_{\text {ext }}^{(n-1)}\right)^{T} \Delta \mathbf{u}^{(n)}+\left(\boldsymbol{\lambda}^{(n)}\right)^{T} \mathcal{R}^{(n)}+\left(\boldsymbol{\mu}^{(n)}\right)^{T} \mathcal{R}^{(n-1)}\right\} .
$$

Due to the asserted static equilibrium the residuals $\mathcal{R}^{(n)}$ and $\mathcal{R}^{(n-1)}$ must vanish. The objective value is thus invariant with respect to the values of the Lagrangian multipliers $\boldsymbol{\lambda}^{(n)}$ and $\boldsymbol{\mu}^{(n)}$ $\left(n=1, \ldots, n_{\text {load }}\right)$, i.e.

$$
\widehat{J}\left(\boldsymbol{\rho} ;\left\{\boldsymbol{\lambda}^{(n)}, \boldsymbol{\mu}^{(n)}\right\}_{n=1, \ldots, n_{\text {load }}}\right)=J(\boldsymbol{\rho}) .
$$

This equivalence also holds for the sensitivity with respect to changes of the topology design variable $\rho_{i}$ on element $i$

$$
\frac{\partial \widehat{J}}{\partial \rho_{i}}=\frac{\partial J}{\partial \rho_{i}}
$$

In the following the derivative $\partial \widehat{J} / \partial \rho_{i}$ is computed with properly determined values of $\boldsymbol{\lambda}^{(n)}$ and $\boldsymbol{\mu}^{(n)}$ leading to simplifications in the derivation. To formally describe these derivations, we introduce a partitioning of all degrees of freedom (DOF) into essential (index E; associated with Dirichlet boundary conditions) and free (index F; remaining DOF) entries. For a vector w and a matrix $\mathbf{M}$ we have

$$
\mathbf{w} \sim\left[\begin{array}{c}
\mathbf{w}_{\mathrm{E}} \\
\mathbf{w}_{\mathrm{F}}
\end{array}\right] \text { and } \mathbf{M} \sim\left[\begin{array}{ll}
\mathbf{M}_{\mathrm{EE}} & \mathbf{M}_{\mathrm{EF}} \\
\mathbf{M}_{\mathrm{FE}} & \mathbf{M}_{\mathrm{FF}}
\end{array}\right] .
$$


In the current context, the displacements $\mathbf{u}_{\mathrm{E}}$ on the Dirichlet boundary are prescribed. They are independent of the current value of $\rho$. This implies that

$$
\frac{\partial \Delta \mathbf{u}}{\partial \rho_{i}}=\frac{\partial}{\partial \rho_{i}}\left[\begin{array}{c}
\Delta \mathbf{u}_{\mathrm{E}} \\
\Delta \mathbf{u}_{\mathrm{F}}
\end{array}\right]=\left[\begin{array}{c}
\mathbf{0} \\
\partial\left\{\Delta \mathbf{u}_{\mathrm{F}}\right\} / \partial \rho_{i}
\end{array}\right]
$$

holds for arbitrary increment loads, i.e. for $\mathbf{u}=\mathbf{u}^{(n)}$ or $\mathbf{u}=\mathbf{u}^{(n-1)}$. The components $\mathbf{f}_{\text {ext,F }}$ of the force vector $\mathrm{f}_{\text {ext }}$ vanish at all times and the only (possibly) non-zero components are the reaction forces $f_{\text {ext, }}$

$$
\mathbf{f}_{\mathrm{ext}}^{(n)}=\left[\begin{array}{c}
\mathbf{f}_{\mathrm{ext}, \mathrm{E}}^{(n)} \\
\mathbf{0}
\end{array}\right]
$$

Eqs. (42) and (43) imply

$$
\left(\mathbf{f}_{\mathrm{ext}}^{(m)}\right)^{T} \frac{\partial \Delta \mathbf{u}^{(n)}}{\partial \rho_{i}}=0
$$

Hence for arbitrary time indices $n, m=1, \ldots, n_{\text {load }}$, we have

$$
\frac{\partial}{\partial \rho_{i}}\left(\left(\mathbf{f}_{\mathrm{ext}}^{(m)}\right)^{T} \Delta \mathbf{u}^{(n)}\right)=\left(\frac{\partial \mathbf{f}_{\mathrm{ext}}^{(m)}}{\partial \rho_{i}}\right)^{T} \Delta \mathbf{u}^{(n)} .
$$

Therefore, the derivative of the modified design objective in (38) can be rewritten as:

$$
\frac{\partial \widehat{J}}{\partial \rho_{i}}=\frac{1}{2} \sum_{n=1}^{n_{\text {load }}}\left\{\left(\frac{\partial \mathbf{f}_{\text {ext }}^{(n)}}{\partial \rho_{i}}+\frac{\partial \mathbf{f}_{\mathrm{ext}}^{(n-1)}}{\partial \rho_{i}}\right)^{T} \Delta \mathbf{u}^{(n)}+\left(\boldsymbol{\lambda}^{(n)}\right)^{T} \frac{\partial \mathcal{R}^{(n)}}{\partial \rho_{i}}+\left(\boldsymbol{\mu}^{(n)}\right)^{T} \frac{\partial \mathcal{R}^{(n-1)}}{\partial \rho_{i}}\right\}
$$

Recall the balance equation at each load time increment in (61), the derivatives of $\mathcal{R}^{(m)}$ at the equilibrium of the $m$-th load increment with respect to $\rho_{i}$ can be expanded as

$$
\frac{\partial \mathcal{R}^{m}}{\partial \rho_{i}}=\frac{\partial \mathbf{f}_{\mathrm{ext}}^{m}}{\partial \rho_{i}}-\int_{\Omega_{i}} \mathbf{B}^{T}\left(\sigma^{e}\right)^{(m)} d \Omega_{i}-\mathbf{K}_{t a n}^{(m)} \frac{\partial \Delta \mathbf{u}^{(m)}}{\partial \rho_{i}}
$$

where

$$
\mathbf{K}_{\mathrm{tan}}^{(m)}=-\frac{\partial \mathcal{R}^{(m)}}{\partial \mathbf{u}^{(m)}}
$$

is the tangent stiffness matrix of the nonlinear mechanical system at the balance equation of the $m$-th load increment. With the expression (47), (46) can be reformulated as

$$
\begin{aligned}
& \frac{\partial \widehat{J}}{\partial \rho_{i}}=\frac{1}{2} \sum_{n=1}^{n_{\text {load }}}\left\{\quad\left(\frac{\partial \mathbf{f}_{\text {ext }}^{(n)}}{\partial \rho_{i}}\right)^{T}\left(\Delta \mathbf{u}^{(n)}+\boldsymbol{\lambda}^{(n)}\right)+\left(\frac{\partial \mathbf{f}_{\text {ext }}^{(n-1)}}{\partial \rho_{i}}\right)^{T}\left(\Delta \mathbf{u}^{(n)}+\boldsymbol{\mu}^{(n)}\right)\right. \\
& -\left(\boldsymbol{\lambda}^{(n)}\right)^{T}\left(\int_{\Omega_{i}} \mathbf{B}^{T}\left(\boldsymbol{\sigma}^{e}\right)^{(n)} d \Omega_{i}+\mathbf{K}_{t a n}^{(n)} \frac{\partial \Delta \mathbf{u}^{(n)}}{\partial \rho_{i}}\right) \\
& \left.-\left(\boldsymbol{\mu}^{(n)}\right)^{T}\left(\int_{\Omega_{i}} \mathbf{B}^{T}\left(\boldsymbol{\sigma}^{e}\right)^{(n-1)} d \Omega_{i}+\mathbf{K}_{t a n}^{(n-1)} \frac{\partial \Delta \mathbf{u}^{(n-1)}}{\partial \rho_{i}}\right)\right\} .
\end{aligned}
$$


As mentioned before, the aim is to find proper values of the Lagrangian multipliers $\boldsymbol{\lambda}^{(n)}$ and $\boldsymbol{\mu}^{(n)}$ such that the sensitivities can be explicitly and efficiently computed. From (43), the first two terms in (49) can be omitted by setting

$$
\boldsymbol{\lambda}_{\mathrm{E}}^{(n)}=-\Delta \mathbf{u}_{\mathrm{E}}^{(n)} \quad \text { and } \quad \boldsymbol{\mu}_{\mathrm{E}}^{(n)}=-\Delta \mathbf{u}_{\mathrm{E}}^{(n)}
$$

Accounting further for the structure of the sensitivities of $\mathbf{u}$ in (42) and for the symmetry of the stiffness matrices we have

$$
\begin{aligned}
& \frac{\partial \widehat{J}}{\partial \rho_{i}}=\frac{1}{2} \sum_{n=1}^{n_{\text {load }}}\left\{\quad-\left(\boldsymbol{\lambda}^{(n)}\right)^{T} \int_{\Omega_{i}} \mathbf{B}^{T}\left(\boldsymbol{\sigma}^{e}\right)^{(n)} d \Omega_{i}-\left(\boldsymbol{\mu}^{(n)}\right)^{T} \int_{\Omega_{i}} \mathbf{B}^{T}\left(\boldsymbol{\sigma}^{e}\right)^{(n-1)} d \Omega_{i}\right. \\
& -\left(\mathbf{K}_{\mathrm{tan}, \mathrm{FE}}^{(n)} \boldsymbol{\lambda}_{E}^{(n)}+\mathbf{K}_{\mathrm{tan}, \mathrm{FF}}^{(n)} \boldsymbol{\lambda}_{F}^{(n)}\right)^{T} \frac{\partial \Delta \mathbf{u}_{\mathrm{F}}^{(n)}}{\partial \rho_{i}} \\
& \left.-\left(\mathbf{K}_{\mathrm{tan}, \mathrm{FE}}^{(n-1)} \boldsymbol{\mu}_{E}^{(n)}+\mathbf{K}_{\mathrm{tan}, \mathrm{FF}}^{(n-1)} \boldsymbol{\mu}_{F}^{(n)}\right)^{T} \frac{\partial \Delta \mathbf{u}_{\mathrm{F}}^{(n-1)}}{\partial \rho_{i}}\right\} .
\end{aligned}
$$

To avoid the evaluation of the unknown derivatives of $\mathbf{u}_{\mathrm{F}}^{(n)}$ and $\mathbf{u}_{\mathrm{F}}^{(n-1)}$, i.e. eliminating the last two lines of (51), the values of $\boldsymbol{\lambda}_{\mathrm{F}}^{(n)}$ and $\boldsymbol{\mu}_{\mathrm{F}}^{(n)}$ are sought as following by solving the adjoint systems with the prescribed values $\lambda_{\mathrm{E}}^{(n)}=-\Delta \mathbf{u}_{\mathrm{E}}^{(n)}$ and $\boldsymbol{\mu}_{\mathrm{E}}^{(n)}=-\Delta \mathbf{u}_{\mathrm{E}}^{(n)}$ at the essential nodes:

$$
\boldsymbol{\lambda}_{\mathrm{F}}^{(n)}=\left(\mathbf{K}_{\mathrm{tan}, \mathrm{FF}}^{(n)}\right)^{-1} \mathbf{K}_{\mathrm{tan}, \mathrm{FE}}^{(i)} \Delta \mathbf{u}_{\mathrm{E}}^{(n)},
$$

and

$$
\boldsymbol{\mu}_{\mathrm{F}}^{(n)}=\left(\mathbf{K}_{\mathrm{tan}, \mathrm{FF}}^{(n-1)}\right)^{-1} \mathbf{K}_{\mathrm{tan}, \mathrm{FE}}^{(n-1)} \Delta \mathbf{u}_{\mathrm{E}}^{(n)} .
$$

The two relations (52) and (53) together with (50) fully determine the values of the Lagrange multipliers $\boldsymbol{\lambda}^{(n)}$ and $\boldsymbol{\mu}^{(n)}$. Finally, the objective function $\partial \widehat{J} / \partial \rho_{i}$ can be computed via

$$
\frac{\partial \widehat{J}}{\partial \rho_{i}}=-\frac{1}{2} \sum_{n=1}^{n_{\text {load }}}\left\{\left(\boldsymbol{\lambda}^{(n)}\right)^{T} \int_{\Omega_{i}} \mathbf{B}^{T}\left(\boldsymbol{\sigma}^{e}\right)^{(n)} d \Omega_{i}+\left(\boldsymbol{\mu}^{(n)}\right)^{T} \int_{\Omega_{i}} \mathbf{B}^{T}\left(\boldsymbol{\sigma}^{e}\right)^{(n-1)} d \Omega_{i}\right\} .
$$

The computation of the sensitivity consists in solving two linear systems of equations (52) and (53). Note that because the proportional loading is increased at a constant rate, i.e.

$$
\Delta \mathbf{u}_{\mathrm{E}}^{(n)}=\frac{\Delta t^{(n)}}{\Delta t^{(n-1)}} \Delta \mathbf{u}_{\mathrm{E}}^{(n-1)}
$$

the solution of the second linear system (53) can also be omitted by means of the recursion formula

$$
\boldsymbol{\mu}_{\mathrm{F}}^{(n)}=\frac{\Delta t^{(n)}}{\Delta t^{(n-1)}} \boldsymbol{\lambda}_{\mathrm{F}}^{(n-1)}
$$

\subsection{Extended BESO method}

The extended BESO method recently developed in [34] using an additional damping treatment on sensitivity numbers is adopted in this work. It has been shown that this treatment can improve the 
robustness and effectiveness of the method, especially in dealing with nonlinear designs in presence of dissipative effects. In this method, the sensitivity numbers associated with the relative ranking of the element sensitivities are chosen to determine material phase exchange. When uniform meshes are used, the sensitivity numbers for the considered objective are defined as following using the element sensitivity computed from (54)

$$
\alpha_{i}=\left\{\begin{array}{cc}
\left(\frac{\partial \widehat{J}}{\partial \rho_{i}}\right)^{\eta}, & \text { for } \rho_{i}=1 \\
0, & \text { for } \rho_{i}=0
\end{array}\right.
$$

in which $\eta$ is a numerical damping coefficient (the same as the one applied in the Optimality Criteria method for density-based methods [63]). When $\eta=1$, we recover the conventional sensitivity numbers for linear elastic designs [64]. In the presence of dissipative effects, the sensitivity numbers vary by several orders of magnitude resulting in instabilities of the topology evolution process, especially when removing certain structural branches. For this reason, the sensitivity numbers are damped in this work with " $\eta=0.5$ " as suggested in $[3,34]$.

To avoid checkerboard patterns, sensitivity numbers are firstly smoothed by means of a filtering scheme [63]

$$
\alpha_{i}=\frac{\sum_{j=1}^{N_{\mathrm{i}}} w_{i j} \alpha_{j}}{\sum_{j=1}^{N_{\mathrm{i}}} w_{i j}},
$$

where $w_{i j}$ is a linear weight factor

$$
w_{i j}=\max \left(0, r_{\min }-\Delta(i, j)\right)
$$

determined according to the prescribed filter radius $r_{\min }$ and the element center-to-center distance $\Delta(i, j)$ between elements $e$ and $j$. We note that the filter (58) is also responsible for material exchange from the matrix phase $\left(\rho_{i}=0\right)$ to the inclusion phase $\left(\rho_{i}=1\right)$ by attributing filtered sensitivity number values to design variables that are associated to the matrix phase.

Due to the discrete nature of the BESO material model, the current sensitivity numbers are needed to be averaged with their historical information to improve the design convergence (see [30])

$$
\alpha_{i}^{(l)} \leftarrow \frac{\left(\alpha_{i}^{(l)}+\alpha_{i}^{(l-1)}\right)}{2}
$$

In this work, the material volume fraction of reinforced inclusion phases is kept constant during the optimization process. Interfaces between different material phases or even the topology of the reinforced inclusion materials will be tailored through the redistribution of the quantitative inclusion phases. The above extended BESO method is adopted to solve the optimization problem by using the modified sensitivity numbers, which account for the whole fracturing process, involving crack nucleation, propagation and interaction until complete failure of the considered heterogeneous materials, so as to improve the fracture resistance of the specimens. 

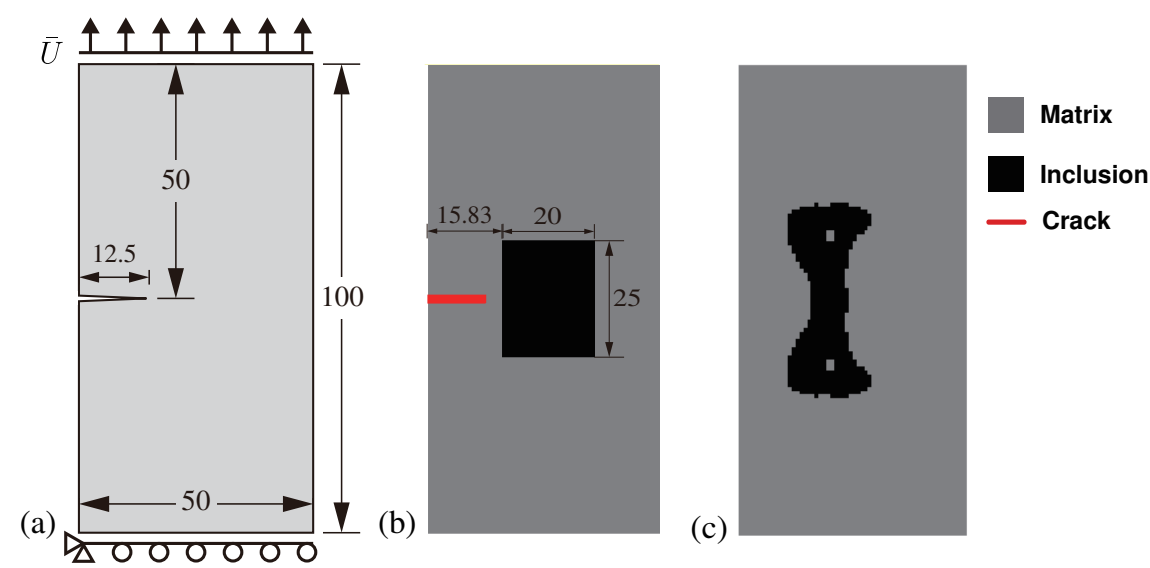

Figure 4. A plate with one pre-existing crack notch subjected to incremental traction loads: (a) problem geometry; (b) initial guess design; (c) final design.

\section{NUMERICAL EXAMPLES}

In this section, several numerical examples are presented to demonstrate the potential of the proposed topology optimization framework. In all tests, regular meshes using quadrilateral bilinear elements are adopted, and plane strain condition is assumed. The same finite element discretization is adopted for both displacement and crack phase fields. The regularization parameter $\ell$ describing the width of smeared crack and interface is chosen as two times the finite element size $\ell=2 \ell_{e}$. The material parameters of each phase are taken as: $E_{i}=52 \mathrm{Gpa}, E_{m}=10.4 \mathrm{Gpa}, v_{i}=v_{m}=0.3$, where the indices $i$ and $m$ correspond to the matrix and inclusion materials, respectively. These parameters are those of a mortar composed of a cement paste (matrix) and sand (inclusion). The toughness is $g_{c}=g_{c}^{I}=1 \times 10^{-4} \mathrm{kN} / \mathrm{mm}$ and the interface fracture strength is chosen as $t_{u}=10^{-2}$ Gpa.

\subsection{Design of a plate with one initial crack under traction}

The problem geometry of this example is depicted in Fig. 4(a). The dimensions of the plate are $50 \times 100 \mathrm{~mm}^{2}$, and the domain is uniformly discretized into $60 \times 120$ square shaped bilinear elements. The boundary conditions are as follows: on the lower end, the vertical displacements are fixed while the horizontal displacements are free, and the left bottom corner node is fixed in both directions. On the upper end, the horizontal displacements are free, while the vertical displacements are prescribed with monotonic displacement increments $\bar{U}=0.005 \mathrm{~mm}$ during the simulation. The incremental loading process continues until the reaction forces is below a prescribed value indicating that the structure is completely broken. It is worth noted that during the crack propagation, interfacial damage can occur and interact with the propagation of the pre-existing matrix crack.

Fig. 4(b) is the initial guess design and consists of a single square inclusion occupying a volume fraction of $10 \%$ of the sample. In all next examples, the material volume fraction of the inclusion phase is maintained constant during the optimization process. By the extended BESO method, the inclusion phase will be redistributed based on their sensitivity numbers so as to improve the fracture resistance of the considered structure. The pre-existing crack notch is simulated by prescribing Dirichlet conditions on the crack phase field with $d=1$ along the crack. The surrounding area of 


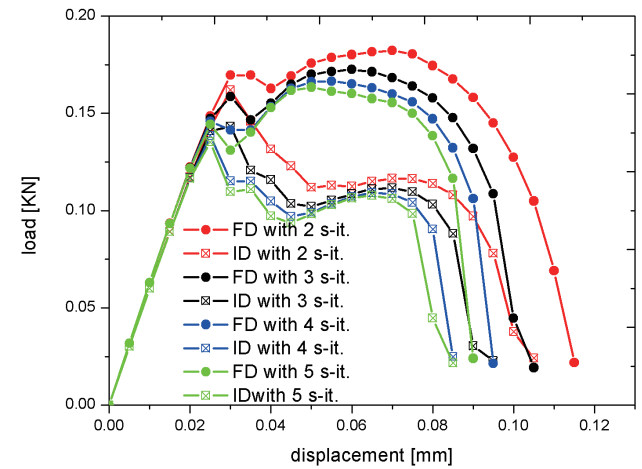

(a)

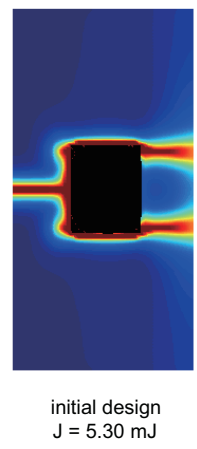

(b)

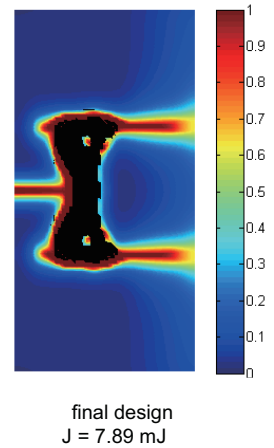

(c)

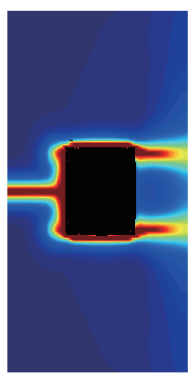

initial design $\mathrm{J}=4.48 \mathrm{~mJ}$

(d)

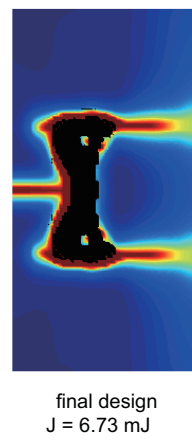

(e)

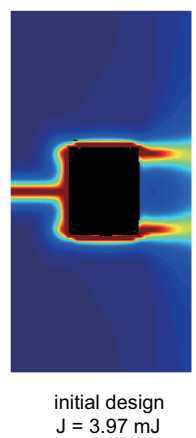

(f)

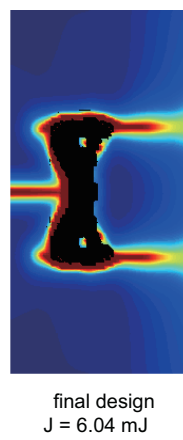

(g)

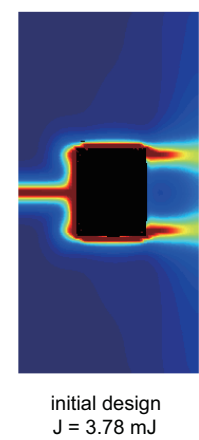

(h)

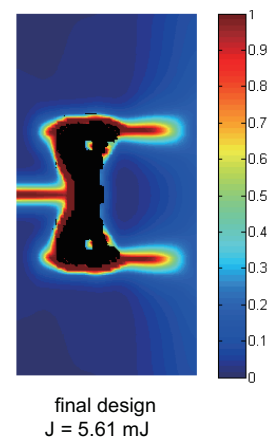

(i)

Figure 5. Fracture resistance comparison of two composite structures with one initial crack subjected to incremental traction loads: (a) load-displacement curves with different numbers of sub-iteration; (b) ID with 2 s-it; (c) FD with 2 s-it; (d) ID with 3 s-it; (e) FD with 3 s-it; (f) ID with 4 s-it; (g) FD with 4 s-it; (h) ID with 5 s-it; (i) FD with 5 s-it.

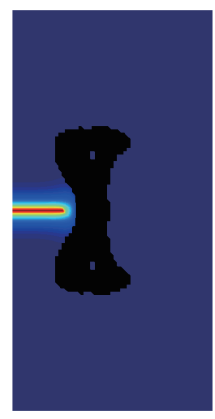

(a)

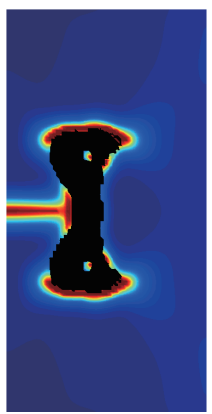

(b)

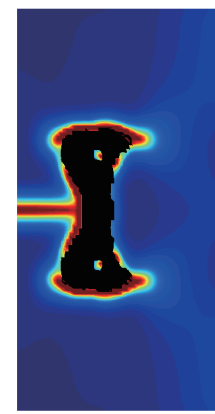

(c)

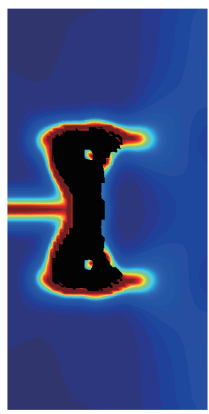

(d)

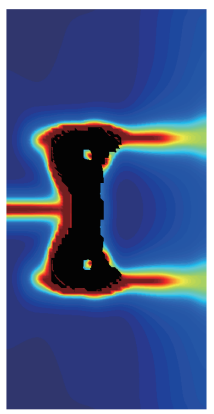

(e)

Figure 6. Crack propagation of the final design composite structure with one initial crack subjected to incremental traction loads: (a) $\bar{U}=0 \mathrm{~mm}$; (b) $\bar{U}=0.05 \mathrm{~mm}$; (c) $\bar{U}=0.06 \mathrm{~mm}$; (d) $\bar{U}=0.08 \mathrm{~mm}$; (e)

$$
\bar{U}=0.095 \mathrm{~mm} \text {. }
$$

the initial crack notch (up to 2 times of the length scale parameter $\ell$ ) is treated a non-designable region to avoid nonphysical designs with the inclusion material added within the already existing crack (see a discussion in [6]). The final structural topology of the inclusion phase is shown in Fig. 4 (c). It can be observed that the material on the right side of the reinforcement inclusion moves up and down on the left side, and holes are generated to tailor the topology of the inclusion phases. The fracture resistance improvement of the resultant composite structure is evaluated by comparing the initial and new designs response in Fig. 5. It is worth noting that in the present work, a staggered 


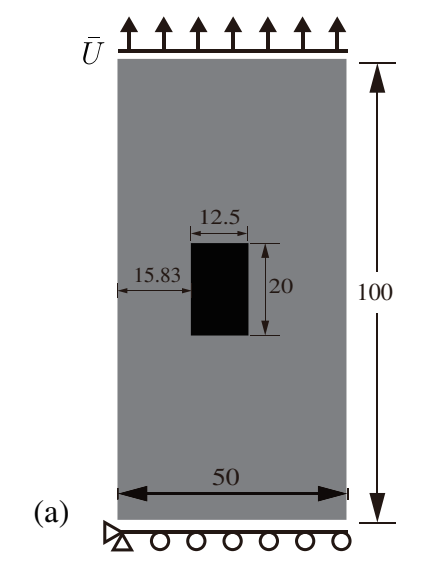

(b)

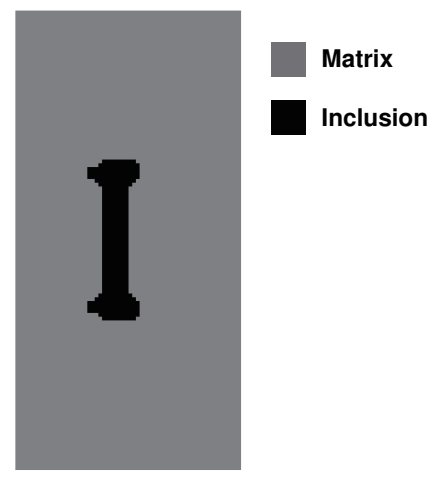

Figure 7. A plate without initial crack subjected to incremental traction loads: (a) geometry of the initial design; (b) final design.

procedure has been employed for solving the coupled displacement-phase field problems formulated in section 2. Then, for one load increment, the number of sub-iterations, i.e. the number of times the displacement and phase field problems are solved alternatively has an effect on the solution. It is worth noting that most authors (see e.g. [16], and also our own previous works, [65, 24], among many others) only use one sub-iteration by assuming that the load increments are small enough, but thus requires a preliminary convergence study. In the following, we have studied the effects of using 2, 3, 4 and 5 sub-iterations in the staggered scheme on the load-displacement response of the structure. In Fig. 5, we denote by "FD" and "ID" Final and Initial Designs, respectively, and "s-it" means sub-iterations. We can observe from Fig. 5 (a) that the number of sub-iterations in the whole optimization procedure does have an effect on the final load-displacement curve but almost no effects on the final optimized shape (see Fig. 5 (b)-(i)). We note that for 4 sub-iterations, the load-displacement curve is roughly converged. With these observations in mind, we then conduct the whole optimization procedure for obtaining the final design with only 2 sub-iterations to reduce the computational costs, as it has been seen that the number of sub-iterations as small influence on the final shape of the inclusion. Then, we use the obtained final design and re-compute the loaddisplacement curve with 4 sub-iterations to avoid underestimating the fracture energy. In the present example, we can see from Fig. 5 that the total required fracture energies for complete failure is 3.97 $\mathrm{mJ}$ for the initial design and $6.04 \mathrm{~mJ}$ for the final design, which means that the final structure is $52 \%$ more resistant to fracture than the initial guess design.

Detailed propagation of the phase field crack of the final design composite structure with one pre-existing crack notch subjected to incremental traction loads is given in Fig. 6. The initial crack propagates into the inner supporting structure and is blocked by the reinforced inclusion phase during the previous incremental loads. Then, two interface cracks nucleate and propagate along the upper and lower material interfaces. Inclusion materials which are redistributed to up and down on the left side try to prevent the vertical propagation of matrix crack. Finally, the interface and matrix cracks are intersected and propagated horizontally until the structure is fully broken. 


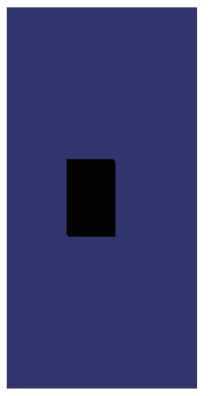

(a)

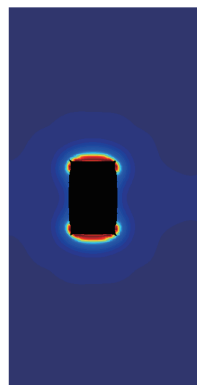

(b)

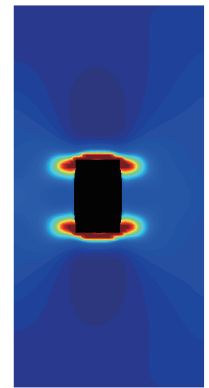

(c)

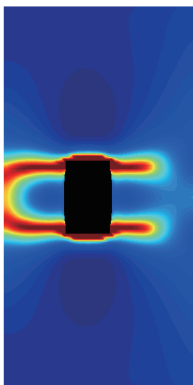

(d)

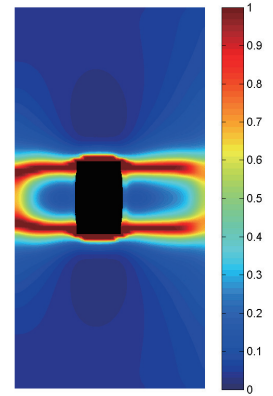

(e)

Figure 8. Crack propagation of the initial design composite structure with one initial crack subjected to incremental traction loads: (a) $\bar{U}=0 \mathrm{~mm}$; (b) $\bar{U}=0.025 \mathrm{~mm}$; (c) $\bar{U}=0.065 \mathrm{~mm}$; (d) $\bar{U}=0.085 \mathrm{~mm}$; (e) $\bar{U}=0.105 \mathrm{~mm}$.

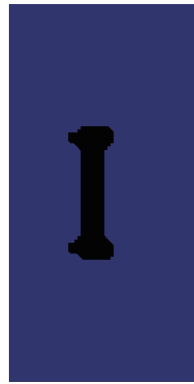

(a)

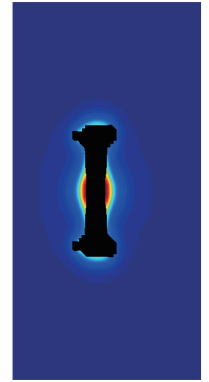

(b)

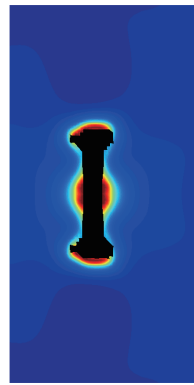

(c)

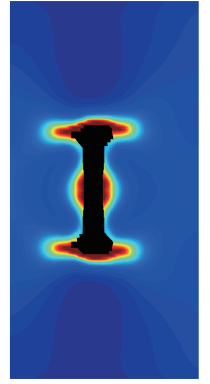

(d)

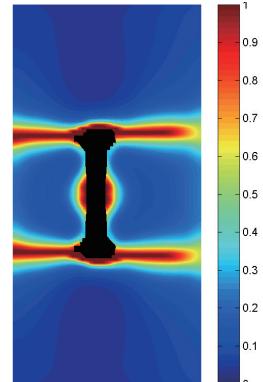

(e)

Figure 9. Crack propagation of the initial design composite structure with one initial crack subjected to incremental traction loads: (a) $\bar{U}=0 \mathrm{~mm}$; (b) $\bar{U}=0.035 \mathrm{~mm}$; (c) $\bar{U}=0.055 \mathrm{~mm}$; (d) $\bar{U}=0.075 \mathrm{~mm}$; (e) $\bar{U}=0.105 \mathrm{~mm}$.

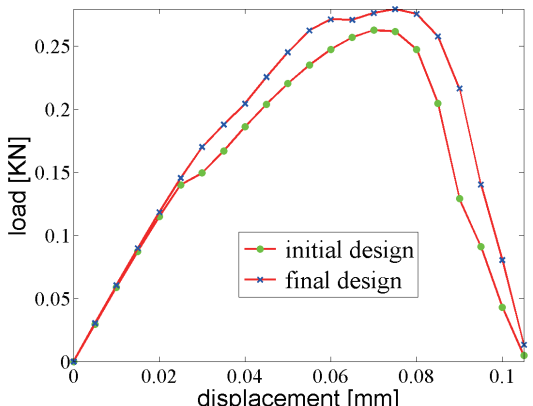

(a)

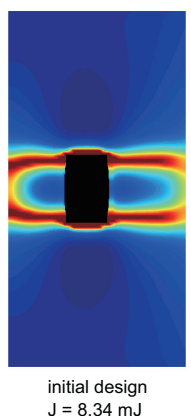

(b)

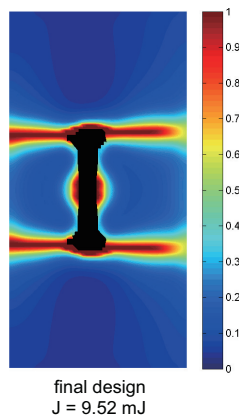

(c)

Figure 10. Fracture resistance comparison of two composite structures without initial crack subjected to incremental traction loads

\subsection{Design of a plate without initial cracks for traction loads}

The problem setting of this example is the same as in the last section except that there is no initial crack. The geometry of the plate is depicted in Fig. 7 (a), where the inclusion phase occupies a volume fraction of $5 \%$ of the sample. The optimized geometry of the inclusion phase is depicted in Fig. 7 (b). Detailed propagations of the phase field cracks of the initially and finally designed composite structures subjected to incremental traction load are given in Fig. 8 and Fig. 9, respectively. The cracks are firstly generated around the upper and lower material interfaces for 


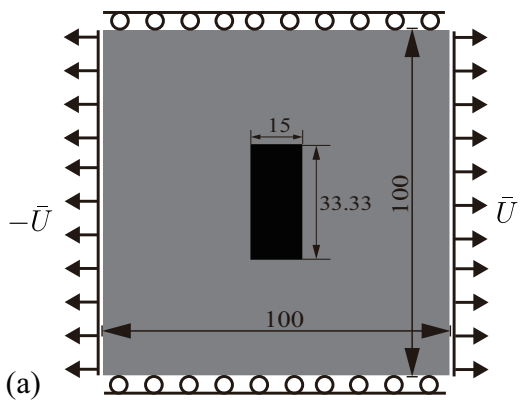

(b)

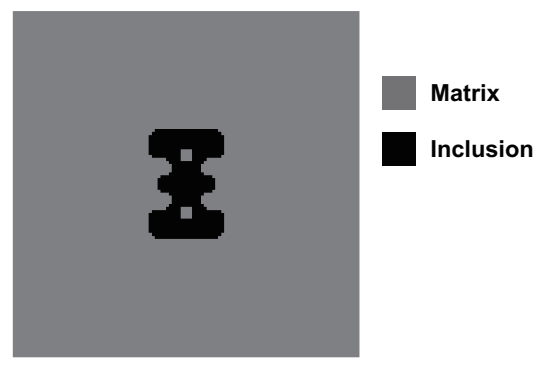

Figure 11. A square plate without initial crack subjected to uniaxial tension: (a) geometry of the initial design; (b) final design.

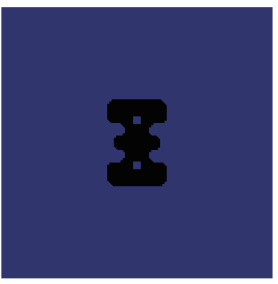

(a)

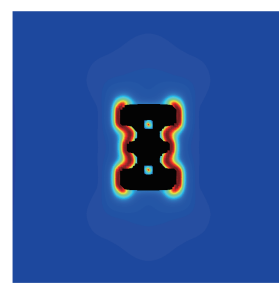

(b)

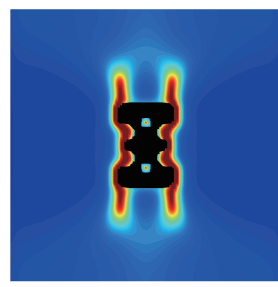

(c)

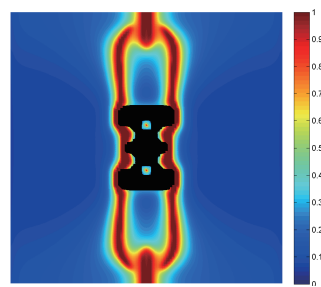

(d)

Figure 12. Crack propagation of the initial design composite structure with one initial crack subjected to uniaxial tension: (a) $\bar{U}=0 \mathrm{~mm}$; (b) $\bar{U}=0.025 \mathrm{~mm}$; (c) $\bar{U}=0.04 \mathrm{~mm}$; (d) $\bar{U}=0.08 \mathrm{~mm}$.

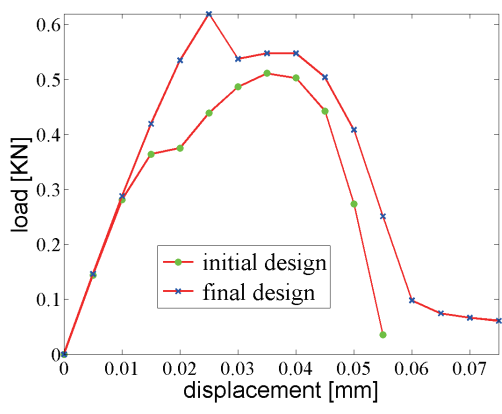

(a)

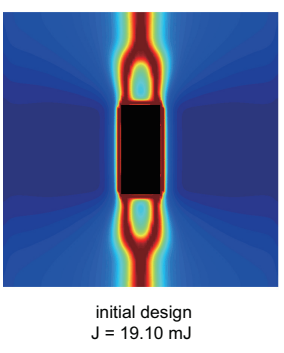

(b)

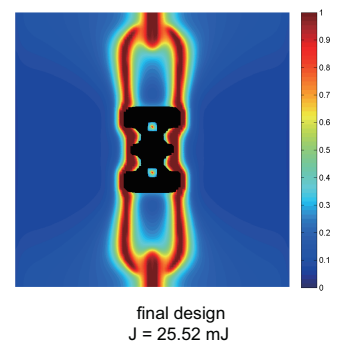

(c)

Figure 13. Fracture resistance comparison of two composite structures without initial crack subjected to uniaxial tension.

the initial guess design, while it nucleates in the middle of the inner inclusion phase as well as in the interfaces for the new design. Then, similar interface cracks around the upper and lower material interfaces are generated. Finally, cracks propagate horizontally until the structure is fully broken in both cases. The final crack patterns, the total required fracture energies for complete failure, and the load-displacement curves for the initial and optimized designs are given in Fig. 10. In this example, the structure with optimal design is $15 \%$ more resistant to fracture than the initial composite structure.

\subsection{Design of a square plate without initial cracks in tensile loading}

This example aims to design a square composite plate without initial crack subjected to uniaxial tension. The problem geometry of the square plate is depicted in Fig. 11 (a). The dimensions 
(a)

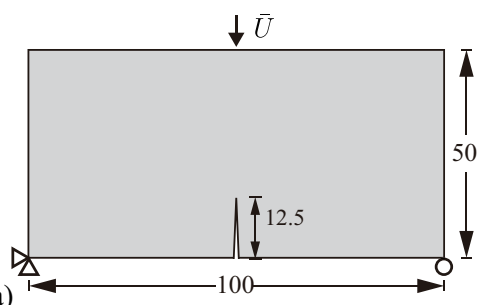

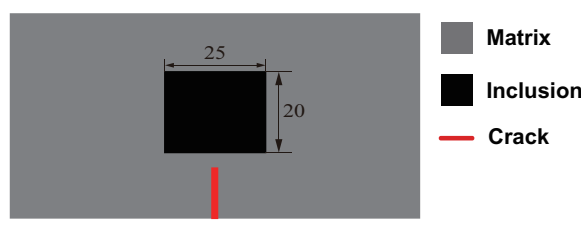

(b)

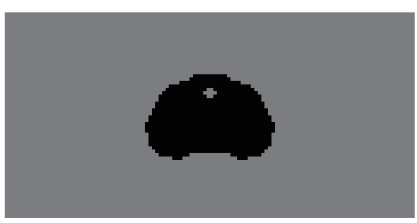

(c)

Figure 14. A plate with one initial crack subjected to three-point bending: (a) geometry and boundary condition; (b) geometry of the initial design and crack; (c) final design.

(a)
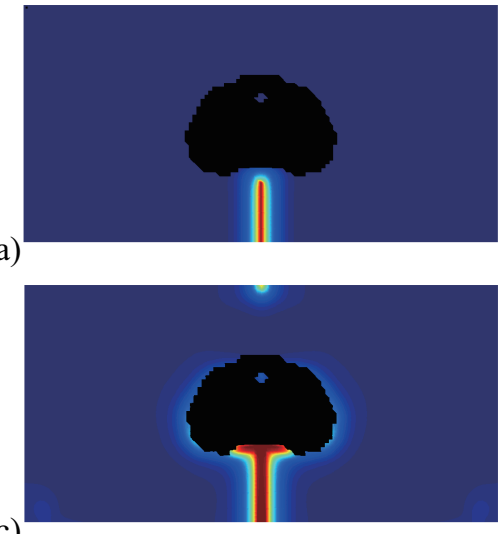

(b)

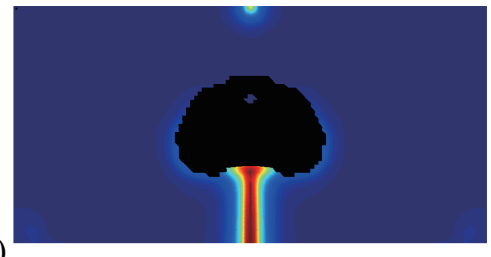

(d)

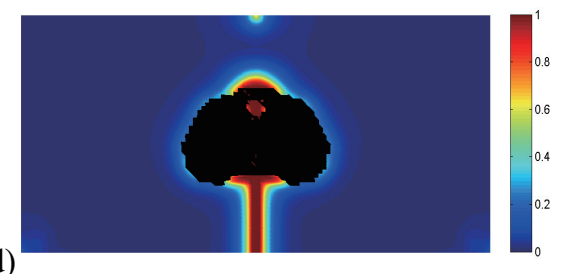

Figure 15. Crack propagation of the initial design composite structure with one initial crack subjected to uniaxial tension: (a) $\bar{U}=0 \mathrm{~mm}$; (b) $\bar{U}=0.11 \mathrm{~mm}$; (c) $\bar{U}=0.14 \mathrm{~mm}$; (d) $\bar{U}=0.16$.

of the plate are $100 \times 100 \mathrm{~mm}^{2}$, and the domain is uniformly discretized into $120 \times 120$ square bilinear elements. The boundary conditions are as follows: on the upper and lower end, the vertical displacements are fixed while the horizontal displacements are free. On the left and right end, the horizontal displacements are prescribed to an increasing uniform value of $\bar{U}=0.005 \mathrm{~mm}$ during the simulation. The incremental loading process continues until the reaction force is below a prescribed value indicating that the structure is fully broken. The initial guess involves an inclusion representing $5 \%$ of the volume fraction as shown in Fig. 11 (a). The final design of the inclusion phase is shown in Fig. 11 (b). The width of the final design is larger than the initial guess to resist the $x$-directional tension, and the inclusion phase topology is changed. Detailed propagation of the phase field crack in the composite structures with optimal design is given in Fig. 12. Here, the interface cracks firstly initiate at the interface (see Fig. 12 (b)). Subsequently, the left and right interface cracks propagate vertically and merge. The complete fracture patterns and design objective values are shown in Fig. 13 (b) for the initial design and in Fig. 13 (c) for the optimal design. Both responses are compared in Fig. 13 (a). Here, the fracture resistance of the final design structure has been increased by $33 \%$ as compared to the initial design. 


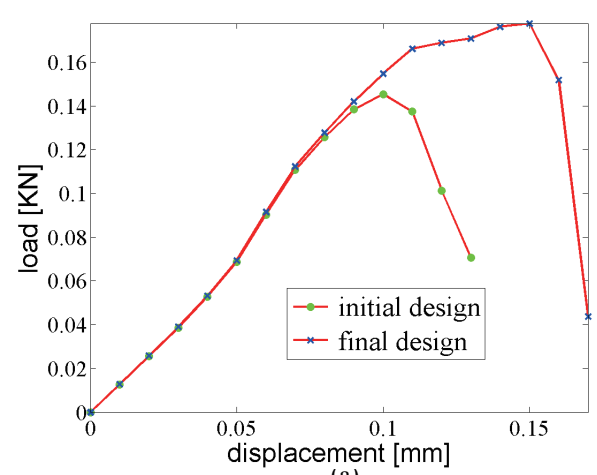

(a)

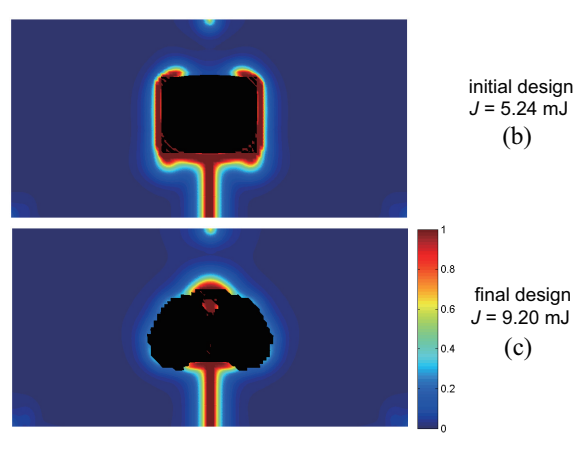

Figure 16. Fracture resistance comparison of two composite structures with one initial crack subjected to three-point bending.

\subsection{Design of a plate with one initial crack in three-point bending}

The purpose of this example is to design a plate subjected to three-point bending with one initial crack. The problem geometry of the square plate is depicted in Fig. 14 (a). The dimensions of the plate are $50 \times 100 \mathrm{~mm}^{2}$. The domain is uniformly discretized into $60 \times 120$ square shape bilinear elements. The load consists into a prescribed displacement at the center of the beam on the top edge. The left bottom corner node is fixed, while node at the right bottom corner the $y$-displacement is fixed and the $x$-displacement is free. For this case, an initial pre-existing crack is shown in Fig. 14 (b), and the initial guess design with the inclusion phase occupying $10 \%$ volume fraction of the domain area. The computation is performed with monotonic displacement increments of $\bar{U}=0.01$ $\mathrm{mm}$ until the reaction forces is below a prescribed criterion value. The displacements are prescribed along the $y$-direction while the displacement along $x$ is free.

The pre-existing crack is simulated by prescribing Dirichlet conditions with $d=1$ along the crack. The surrounding area of the initial crack notch is treated as a non-designed region. Fig. 14 (c) shows the final design of inclusion topology. Detailed propagation of the phase field crack of the finally designed composite structure with one pre-existing crack notch subjected to three-point bending with monotonic displacement increments is shown in Fig. 15. The initial matrix crack firstly propagates vertically and is blocked by the reinforced inclusion phase materials. During the next steps, it tries to spread along the horizontal direction but is blocked by the inclusion material redistributed by using the proposed topological optimization method. Eventually, the matrix crack propagates along the loading direction until the structure is fully broken. The two load-displacement curves are compared in Fig. 16 (a). The final crack patterns as well as the total required fracture energies for complete failure for the initial and final designs are shown in Fig. 16 (b) and Fig. 16 (c), respectively. In this example, the fracture resistance of the structure with final design has been increased by $76 \%$ as compared to the initial design.

\subsection{Design of a plate containing multiple inclusions}

Finally, a plate containing 20 periodically distributed square inclusions is considered. The plate is modeled as a square domain whose length is $100 \mathrm{~mm}$. The length of the inclusions is computed such that the volume fraction of inclusion phase is equal to $20 \%$. The boundary conditions are the same as described in Section 4.3 and is shown in Fig. 17 (a): on the lower and upper ends, the 

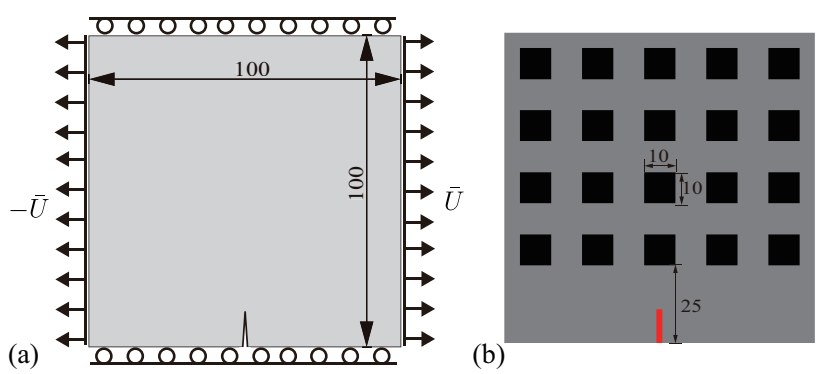

(b)

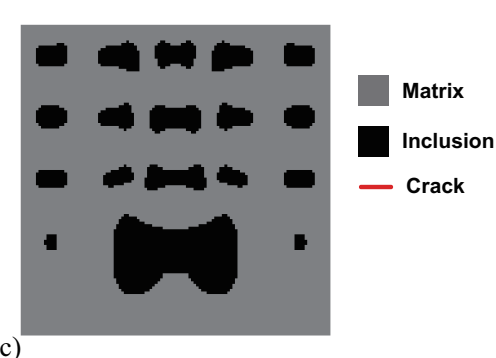

Figure 17. A plate containing multiple inclusions and one initial crack for tensile loads: (a) geometry and boundary condition; (b) geometry of the initial design and crack; (c) final design.
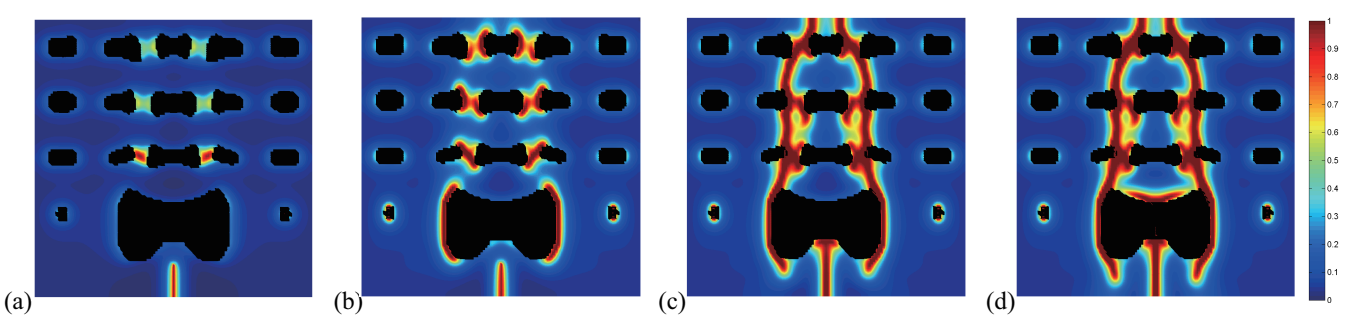

Figure 18. Crack propagation of the final design composite structure with one initial crack subjected to uniaxial tension: (a) $\bar{U}=0.01 \mathrm{~mm}$; (b) $\bar{U}=0.02 \mathrm{~mm}$; (c) $\bar{U}=0.05 \mathrm{~mm}$; (d) $\bar{U}=0.09 \mathrm{~mm}$.

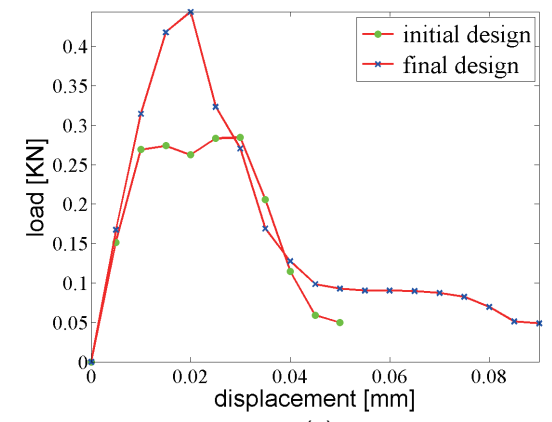

(a)

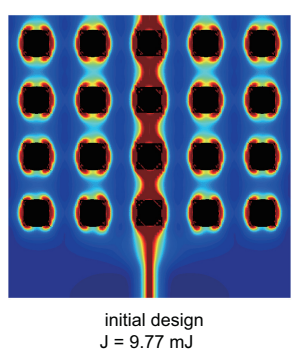

(b)

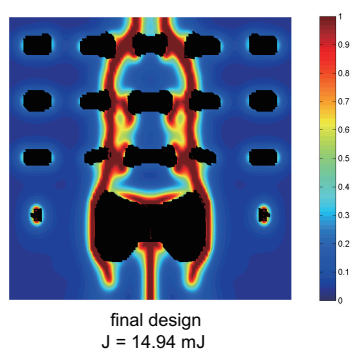

(c)

Figure 19. Fracture resistance comparison of two composite structures containing multiple inclusions and one initial crack subjected to tensile loads

$y$-displacements are fixed, while the $x$-displacements are free. On the left and right ends, the $y$ displacements are free, while $x$-displacements are prescribed, with a uniform increasing value of $\bar{U}$ during the simulation. The computation is performed with monotonic displacement increments $\bar{U}=0.005 \mathrm{~mm}$ until the structure is complete broken. Here again, the surrounding region of the initial crack is assumed to be non-designable to avoid nonphysical designs. The final design is presented in Fig. 17 (c). Detailed propagation of the phase field crack of the final design of the considered composite structure containing multiple inclusions and one initial crack subjected to tensile loads is shown in Fig. 18. It can be observed that the initial crack propagates vertically and new cracks around intermediate interfaces of the structure are generated. The inclusion materials are redistributed to prevent further propagation of critical cracks. In the subsequent loading steps, the pre-existing crack meets the reinforced inclusion materials and is blocked at the beginning. Then, both matrix and interface cracks propagate toward the vertical direction as well as along the material 
interfaces until the structure is fully broken. The comparisons of final crack paths, the total required fracture energies for complete failure and load-displacement curves between initial and optimal designs are provided in Fig. 19. In this example, the resistance to fracture has been increased by $53 \%$ in comparison with the initial design.

It is worth noting that in such optimization process, the crack pattern can involve multiple cracks, whose trajectories and number fully depends on the geometry of the inclusion phases. Then, it is very difficult to guess a priori the optimal geometry of inclusions to increase the fracture resistance. Finally, the main advantages of the phase field method in topological optimization process are summarized as follows: (a) it is easy to couple the phase field method with a topological optimization algorithm as a fixed mesh can be employed for the fracture simulation; (b) initiation of cracks can be included in the analysis, which would be not possible with techniques like XFEM [11]; (c) including interfacial damage is simple in the present framework, using e.g. extensions of the phase field as proposed in [24]. Using cohesive elements in that case would make the analysis much more complex when defining the sensitivity with respect to the material density in the topological algorithm. Then, the present framework seems to be very promising to design new composite materials with enhanced fracture resistance.

\section{CONCLUSION}

In this work, a topology optimization framework for improving fracture resistance of two-phase composites through a redistribution of reinforced inclusion phases, considering interactions between bulk brittle fracture and interfacial damage, has been developed. One particular merit of the adopted phase field method is the regularized description of discontinuous fields, which avoids the burden of remeshing during the crack propagation which is fully adapted to topology optimization. The sole and unique phase field is employed to describe both bulk brittle fracture and interface cracking, and thus allows interaction between the two types of cracks. Before performing the topology optimization, a computationally efficient adjoint sensitivity formulation is derived to account for the whole fracturing process, involving cracks nucleation, propagation and interaction, either from the interfaces and then through the solid phases, or the composite. Based on the determined sensitivity numbers, the constant amount of reinforced inclusion materials are redistributed by using the extended bi-directional evolutionary structural optimization method. Several benchmark tests have been presented to demonstrate the potential of the proposed design framework. It has been shown that significant improvement of the fracture resistance of the considered composite structures can be achieved for final designs accounting for full failure when compared to the initial guess designs. To our best knowledge, the topology optimization for fracture resistance taking into account interactions between interfacial damage and bulk brittle fracture for complete fracturing process has been done for the first time in this work. The presented method then provides a very promising design tool to improve the fracture resistance of heterogeneous materials where both interfacial damage and matrix crack propagation occur, and could constitute a basis for improving other physical properties involving interfacial damages. 


\section{ACKNOWLEDGEMENTS}

This work was supported by State Key Program of National Natural Science Foundation of China (61232014) and Institut Universitaire de France (IUF). L. Xia thanks the financial support of the National Natural Science Foundation of China (51705165, 51790171, 5171101743 ).

\section{APPENDIX}

\subsection{Linearization of the displacement problem}

Even though the phase field problem is linear in the staggered scheme, i.e. for a fixed value to $\mathbf{u}$, it should be mentioned that for a fixed crack phase field value $d$, the mechanical problem (25) is nonlinear since the computation of eigenvalues of $\varepsilon^{e}$ in (13) and the interface cohesive model in (31). A linear procedure to solve this nonlinear problem by the Newton method is introduced in the following. From (25) and (29), the balance equation can be rewritten as

$$
\mathcal{R}=\int_{\Omega} \boldsymbol{\sigma}^{e}: \boldsymbol{\varepsilon}^{e}(\delta \mathbf{u}) d \Omega+\int_{\Omega} \gamma_{\beta}(\mathbf{x}) \mathbf{t}(\mathbf{w}, \alpha) \cdot \delta \mathbf{w} d \Omega-\int_{\Omega} \mathbf{f} \cdot \delta \mathbf{u} d \Omega-\int_{\partial \Omega_{F}} \overline{\mathbf{F}} \cdot \mathbf{u} d \Gamma=0,
$$

where $\varepsilon^{e}(\delta \mathbf{u})=\nabla^{s} \delta \mathbf{u}-\mathbf{n} \otimes{ }^{S} \mathbf{w} \gamma_{\beta}$. In a standard Newton method, the displacements are updated for each loading by solving the following tangent equation:

$$
D_{\Delta u} \mathcal{R}\left(\mathbf{u}^{k}, d\right)=-\mathcal{R}\left(\mathbf{u}^{k}, d\right)=0,
$$

where $\mathbf{u}^{k}$ is the displacement solution from the $k$-th iteration. The displacements at the current iteration are given by

$$
\mathbf{u}^{k+1}=\mathbf{u}^{k}+\Delta \mathbf{u}
$$

From (62), we obtain

$$
D_{\Delta \mathbf{u}} \mathcal{R}\left(\mathbf{u}^{k}\right)=\int_{\Omega} \frac{\partial \boldsymbol{\sigma}^{e}}{\partial \varepsilon^{e}}: \varepsilon^{e}(\Delta \varepsilon): \varepsilon^{e}(\delta \varepsilon)+\int_{\Omega} \frac{\partial \mathbf{t}(\mathbf{w})}{\partial \mathbf{w}}: \Delta \mathbf{w}: \delta \mathbf{w} d \Omega
$$

with

$$
\Delta \mathbf{w}(\mathbf{x})=h \nabla \Delta \mathbf{u}(\mathbf{x}) \frac{\nabla \phi(\mathbf{x})}{\|\nabla \phi(\mathbf{x})\|}
$$

and

$$
\frac{\partial\left[\boldsymbol{\sigma}^{e}\right]}{\partial\left[\boldsymbol{\varepsilon}^{e}\right]}=\mathbf{C}(\mathbf{u}, d)=\left[(1-d)^{2}+k\right]\left\{\lambda R^{+}[\mathbf{1}]^{T}[\mathbf{1}]+\mathbf{2} \mu \mathbf{P}^{+}\right\}+\left\{\lambda R^{-}[\mathbf{1}]^{\mathbf{T}}[\mathbf{1}]+\mathbf{2} \mu \mathbf{P}^{-}\right\}
$$

where $\left[\boldsymbol{\sigma}^{e}\right]$ and $\left[\varepsilon^{e}\right]$ are the vector forms for the second-order tensors $\boldsymbol{\sigma}^{e}$ and $\boldsymbol{\varepsilon}^{e}$, respectively and $\mathbf{C}$ is the matrix form corresponding to the fourth-order tensor $\mathbb{C}$.

\subsection{FEM discretization of the phase field problem}

A staggered solution procedure is adopted in this work, where the phase field and the mechanical problems are solved alternatively. At each increment, given the displacement field from the mechanical problem, the phase field problem is linear using a shifted algorithm (see more details in [65]). Using FEM, the phase field and phase field gradient in one element are approximated by:

$$
d(\mathbf{x})=\mathbf{N}_{d}(\mathbf{x}) \mathbf{d}^{e}, \nabla d(\mathbf{x})=\mathbf{B}_{d}(\mathbf{x}) \mathbf{d}^{e}
$$


where $\mathbf{d}_{e}$ are nodal phase field values in one element, $\mathbf{N}_{d}(\mathbf{x})$ and $\mathbf{B}_{d}(\mathbf{x})$ are matrices of shape functions and of shape functions derivatives associated to phase field variable, respectively. Introducing the above FEM discretization into the weak form (24), the following linear discrete system of equations can be obtained:

$$
\mathbf{K}_{d} \tilde{\mathbf{d}}=\mathbf{F}_{d}
$$

where

$$
\mathbf{K}_{d}=\int_{\Omega}\left\{\left(\frac{g_{c}}{\ell}(1-\beta)+2 \mathcal{H}\right) \mathbf{N}_{d}^{\mathbf{T}} \mathbf{N}_{d}+(1-\beta) g_{c} \ell \mathbf{B}_{d}^{T} \mathbf{B}_{d}\right\} d \Omega
$$

and

$$
\mathbf{F}_{d}=\int_{\Omega} 2 \mathbf{N}_{d}^{T} \mathcal{H}\left(\mathbf{u}_{n}\right) d \Omega
$$

\subsection{FEM discretization of the displacement problem}

Similarly, the displacement field and incremental displacement field can be expressed using the FEM approximations

$$
\mathbf{u}=\mathbf{N} \mathbf{u}^{e}, \Delta \mathbf{u}=\mathbf{N} \Delta \mathbf{u}^{e}
$$

where $\mathbf{N}$ denotes the matrix of shape functions associated to displacement variables, $\mathbf{u}^{e}$ and $\Delta \mathbf{u}^{e}$ are nodal displacement components and nodal incremental displacement components in one element. Furthermore, we have

$$
[\varepsilon](\Delta \mathbf{u})=\mathbf{B}_{u} \Delta \mathbf{u}^{e}
$$

where $\mathbf{B}_{u}$ is a matrix of shape function derivatives. From (7), the diffuse jump approximation vector and its incremental counterparts can be discretized as

$$
\mathbf{w}=h \mathbf{N} \tilde{\mathbf{B}}_{u} \mathbf{u}^{e}, \Delta \mathbf{w}=h \mathbf{N} \tilde{\mathbf{B}}_{u} \Delta \mathbf{u}^{e}
$$

where

$$
\mathbf{N}=\left[\begin{array}{cccc}
n_{1} & n_{2} & 0 & 0 \\
0 & 0 & n_{1} & n_{2}
\end{array}\right]
$$

and $n_{1}$ and $n_{2}$ are the $x$ - and $y$ - components of the normal vector. The smoothed jump strain at the interfaces is defined by

$$
[\bar{\varepsilon}]=\left[\begin{array}{c}
\bar{\varepsilon}_{11} \\
\bar{\varepsilon}_{22} \\
\sqrt{2} \bar{\varepsilon}_{12}
\end{array}\right]=\gamma_{\beta}(\mathbf{x})\left[\begin{array}{c}
\omega_{1} n_{1} \\
\omega_{2} n_{2} \\
\frac{1}{\sqrt{2}}\left(\omega_{1} n_{2}+\omega_{2} n_{1}\right)
\end{array}\right] .
$$

Then it yields

$$
[\bar{\varepsilon}(\Delta u)]=h \gamma_{\beta}(\mathbf{x}) \mathbf{M} \tilde{\mathbf{B}}_{\mathbf{u}} \Delta \mathbf{u}^{\mathbf{e}}
$$

with

$$
\mathbf{M}=\left[\begin{array}{cccc}
n_{1}^{2} & n_{1} n_{2} & 0 & 0 \\
0 & 0 & n_{1} n_{2} & n_{2}^{2} \\
\frac{1}{\sqrt{2}} n_{1} n_{2} & \frac{1}{\sqrt{2}} n_{2}^{2} & \frac{1}{\sqrt{2}} n_{1}^{2} & \frac{1}{\sqrt{2}} n_{1} n_{2}
\end{array}\right]
$$

With the above FEM discretization, the linear tangent problem reduces to the following linear system of algebraeic equations

$$
\mathbf{K}_{\tan } \Delta \tilde{\mathbf{u}}=-\mathbf{R}\left(\tilde{\mathbf{u}}^{k}\right)
$$

where

$$
\mathbf{K}_{t a n}=\int_{\Omega}\left[\mathbf{B}_{u}^{T}-h \gamma_{\beta}(\mathbf{x}) \tilde{B}_{u}^{T} \mathbf{M}^{T}\right] \mathbf{C}(\mathbf{x})\left[\mathbf{B}_{u}-h \gamma_{\beta}(\mathbf{x}) \tilde{B}_{u} \mathbf{M}\right] d \Omega+\int_{\Omega} h^{2} \gamma_{\beta}(\mathbf{x}) \tilde{B}_{u}^{T} \mathbf{N}^{T} \mathbf{K}_{I} \mathbf{N} \tilde{B}_{u} d \Omega
$$


and

$$
\begin{aligned}
\mathbf{R}= & \int_{\Omega}\left[\mathbf{B}_{u}^{T}-h \gamma_{\beta}(\mathbf{x}) \tilde{\mathbf{B}}_{u}^{T} \mathbf{M}^{T}\right] \mathbf{C}(\mathbf{x})\left[\mathbf{B}_{u}-h \gamma_{\beta}(\mathbf{x}) \tilde{B}_{u} \mathbf{M}\right]\left(\mathbf{u}^{e}\right)^{k} d \Omega \\
& +\int_{\Omega} h \gamma_{\beta}(\mathbf{x}) \tilde{B}_{u}^{T} \mathbf{N}^{T} \mathbf{t}\left(\mathbf{w}^{k}\right) d \Omega+\int_{\Omega} \mathbf{f} \mathbf{N}^{T} d \Omega+\int_{\Omega} \overline{\mathbf{F}} \mathbf{N}^{T} d \Gamma
\end{aligned}
$$

6.3.1. Overall numerical algorithm for crack propagation The overall algorithm is described as follows.

1. Set the initial displacement field $\mathbf{u}_{0}(\mathbf{x})$, the phase field $d_{0}(\mathbf{x})$, and the strain-history function $\mathcal{H}_{0}$.

2. Compute the phase field $\beta(\mathbf{x})$.

3. For all loading increments: (at each time $t_{n+1}$ ), given $d_{n}, \mathbf{u}_{n}$, and $\mathcal{H}_{n}(\mathbf{x})$.

(a) Compute the history function $\mathcal{H}\left(t_{n+1}\right)$ according to (21).

(b) Compute the crack phase field $d_{n+1}(\mathbf{x})$ by solving linear problem (68).

(c) Compute $\mathbf{u}_{n+1}(\mathbf{x})$ :
i. Initialize $\mathbf{u}_{k}=\mathbf{u}_{n}$ While $\left\|\Delta \mathbf{u}_{k+1}\right\|>\epsilon, \epsilon \ll 1$ :
ii. Compute $\Delta \mathbf{u}_{k+1}^{e}$ by (78).
iii. Update $\mathbf{u}_{k+1}=\mathbf{u}_{k}+\Delta \mathbf{u}_{k+1}^{e}$.
iv. $(.)_{n+1} \rightarrow(.)_{n}$ and go to (a).

\section{End}

\section{End}

\section{REFERENCES}

1. Quan Z, Larimore Z, Wu A, Yu J, Qin X, Mirotznik M, Suhr J, Byun JH, Oh Y, Chou TW. Microstructural design and additive manufacturing and characterization of $3 \mathrm{~d}$ orthogonal short carbon fiber/acrylonitrile-butadiene-styrene preform and composite. Composites Science and Technology 2016; 126:139-148.

2. Cadman JE, Zhou S, Chen Y, Li Q. On design of multi-functional microstructural materials. Journal of Materials Science 2013; 48(1):51-66.

3. Fritzen F, Xia L, Leuschner M, Breitkopf P. Topology optimization of multiscale elastoviscoplastic structures. International Journal for Numerical Methods in Engineering 2016; 106(6):430-453.

4. Gu GX, Dimas L, Qin Z, Buehler MJ. Optimization of composite fracture properties: Method, validation, and applications. Journal of Applied Mechanics 2016; 83(7):071 006.

5. San B, Waisman H. Optimization of carbon black polymer composite microstructure for rupture resistance. Journal of Applied Mechanics 2016; 84(2):021 005.

6. Xia L, Da DC, Yvonnet J. Topology optimization for maximizing the fracture resistance of quasi-brittle composites. Computer Methods in Applied Mechanics and Engineering 2018; 332:234-254.

7. Lamon J, Carrere N, Martin E. The influence of the interphase and associated interfaces on the deflection of matrix cracks in ceramic matrix composites. Composites A 2000; 31:1179-1190.

8. Tvergaard V. Model studies of fibre breakage and debonding in a metal reinforced by short fibres. Journal of the Mechanics and Physics of Solids 1993; 41:1309-1326.

9. Nguyen TT, Yvonnet J, Bornert M, Chateau C. Initiation and propagation of complex 3D networks of cracks in heterogeneous quasi-brittle materials: Direct comparison between in situ testing-microCT experiments and phase field simulations. Journal of the Mechanics and Physics of Solids 2016; 99:320-350.

10. Narducci F, Pinho ST. Exploiting nacre-inspired crack deflection mechanisms in cfrp via micro-structural design. Composites Science and Technology 2017; doi:10.1016/j.compscitech.2017.08.023.

11. Moes N, Dolbow J, Belytschko T. A finite element method for crack growth without remeshing. International Journal for Numerical Methods in Engineering 1999; 46:131-150. 
12. Sukumar N, Moes B Nand Moran. Extended finite element method for three-dimensional crack modelling. International Journal for Numerical Methods in Engineering 2000; 48:1549-1570.

13. Bernard PE, Moes N, Chevaugeon N. Damage growth modeling using the thick level set (TLS) approach: Efficient discretization for quasi-static loadings. Computer Methods in Applied Mechanics and Engineering 2012; 233:1127.

14. Cazes F, Moes N. Damage growth modeling using the thick level set (TLS) approach: Efficient discretization for quasi-static loadings. International Journal for Numerical Methods in Engineering 2015; 103(2):114-143.

15. Francfort GA, Marigo JJ. Revisiting brittle fracture as an energy minimization problem. Journal of the Mechanics and Physics of Solids 1998; 46(8):1319-1342.

16. Miehe C, Hofacker M, Welschinger F. A phase field model for rate-independent crack propagation: Robust algorithmic implementation based on operator splits. Computer Methods in Applied Mechanics and Engineering 2010; 199(45-48):2765-2778.

17. Kuhn C, Müller R. A continuum phase field model for fracture. Engineering Fracture Mechanics 2010; 77(18):3625-3634.

18. Bourdin B, Francfort GA, Marigo JJ. The variational approach to fracture. Journal of elasticity 2008; 91(1-3):5148.

19. Pham K, Marigo JJ. The variational approach to damage: I. the foundations. C. R. Mecanique 2010; 338:191-198.

20. Bourdin B, Francfort GA, Marigo JJ. Numerical experiments in revisited brittle fracture. J. Mech. Phys. Solids 2000; 48:797-826.

21. Mumford D, Shah J. Optimal approximations by piecewise smooth functions and associated variational problems. Communications on Pure and Applied Mathematics 1989; 42(5):577-685.

22. Ambrosio L, Tortorelli VM. Approximation of functional depending on jumps by elliptic functional via $\gamma$ convergence. Communications on Pure and Applied Mathematics 1990; 43(8):999-1036.

23. Miehe C, Welschinger F, Hofacker M. Thermodynamically consistent phase-field models of fracture: Variational principles and multi-field fe implementations. International Journal for Numerical Methods in Engineering 2010; 83:1273-1311.

24. Nguyen TT, Yvonnet J, Zhu QZ, Bornert M, Chateau C. A phase-field method for computational modeling of interfacial damage interacting with crack propagation in realistic microstructures obtained by microtomography. Computer Methods in Applied Mechanics and Engineering 2016; 312:567-595.

25. Bendsøe MP, Kikuchi N. Generating optimal topologies in structural design using a homogenization method. Computer Methods in Applied Mechanics and Engineering 1988; 71(2):197-224.

26. Bendsøe M, Sigmund O. Topology Optimization: Theory, Methods and Applications. Springer-Verlag: Berlin, 2003.

27. Nguyen-Xuan H. A polytree-based adaptive polygonal finite element method for topology optimization. Int. J. Numer. Meth. Eng. 2017; 110(10):972-1000.

28. Chau K, Chau K, Ngo T, Hackl K, Nguyen-Xuan H. A polytree-based adaptive polygonal finite element method for multi-material topology optimization. Computer Methods in Applied Mechanics and Engineering 2018; .

29. Xie YM, Steven GP. A simple evolutionary procedure for structural optimization. Computers and Structures 1993; 49(5):885-896.

30. Huang X, Xie YM. Convergent and mesh-independent solutions for the bi-directional evolutionary structural optimization method. Finite Elements in Analysis and Design 2007; 43(14):1039-1049.

31. Sethian JA, Wiegmann A. Structural boundary design via level set and immersed interface methods. Journal of Computational Physics 2000; 163(2):489-528.

32. Wang MY, Wang X, Guo D. A level set method for structural topology optimization. Computer Methods in Applied Mechanics and Engineering 2003; 192(1-2):227-246.

33. Allaire G, Jouve F, Toader AM. Structural optimization using sensitivity analysis and a level-set method. Journal of Computational Physics 2004; 194(1):363-393.

34. Xia L, Fritzen F, Breitkopf P. Evolutionary topology optimization of elastoplastic structures. Structural and Multidisciplinary Optimization 2017; 55(2):569-581.

35. Xia L, Xia Q, Huang X, Xie Y. Bi-directional evolutionary structural optimization on advanced structures and materials: A comprehensive review. Archives of Computational Methods in Engineering 2017; doi:10.1007/ s11831-016-9203-2.

36. Xia L, Breitkopf P. Concurrent topology optimization design of material and structure within $\mathrm{FE}^{2}$ nonlinear multiscale analysis framework. Computer Methods in Applied Mechanics and Engineering, 2014; 278:524-542.

37. Xia L, Breitkopf P. Multiscale structural topology optimization with an approximate constitutive model for local material microstructure. Computer Methods in Applied Mechanics and Engineering, 2015; 286:147-167.

38. Vermaak N, Michailidis G, Parry G, Estevez R, Allaire G, Brechet Y. Material interface effects on the topology optimziation of multi-phase structures using a level set method. Structural and Multidisciplinary Optimization 
2014; doi:10.1007/s00158-014-1074-2.

39. Lawry M, Maute K. Level set topology optimization of problems with soliding contact interfaces. Structural and Multidisciplinary Optimization 2015; 52:1107-1119.

40. Liu P, Luo Y, Kang Z. Multi-material topology optimization considering interface behaviour via xfem and level set method. Computer Methods in Applied Mechanics and Engineering 2016; 308:113-133.

41. Behrou R, Lawry M, Maute K. Level set topology optimization of structural problems with interface cohesion. International Journal for Numerical Methods in Engineering ; doi:10.1002/nme.5540.

42. Duysinx P, Bendsøe MP. Topology optimization of continuum structures with local stress constraints. International Journal for Numerical Methods in Engineering 1998; 43(8):1453-1478.

43. Guo X, Zhang WS, Wang MY, Wei P. Stress-related topology optimization via level set approach. Computer Methods in Applied Mechanics and Engineering 2011; 200(47-48):3439-3452.

44. Xia Q, Shi T, Liu S, Wang MY. A level set solution to the stress-based structural shape and topology optimization. Computers and Structures 2012; 90-91:55-64.

45. Bruggi M, Duysinx P. Topology optimization for minimum weight with compliance and stress constraints. Structural and Multidisciplinary Optimization 2012; 46(3):369-384.

46. Luo Y, Wang MY, Kang Z. An enhanced aggregation method for topology optimization with local stress constraints. Computer Methods in Applied Mechanics and Engineering 2013; 254:31-41.

47. Cai S, Zhang W, Zhu J, Gao T. Stress constrained shape and topology optimization with fixed mesh: A b-spline finite cell method combined with level set function. Computer Methods in Applied Mechanics and Engineering 2014; 278:361-387.

48. Cai S, Zhang W. Stress constrained topology optimization with free-form design domains. Computer Methods in Applied Mechanics and Engineering 2015; 289:267-290.

49. Sun Z, Li D, Zhang WS, Shi S, Guo X. Topological optimization of biomimetic sandwich structures with hybrid core and cfrp face sheets. Composites Science and Technology 2017; 142:79-90.

50. Challis VJ, Roberts AP, Wilkins A. Fracture resistance via topology optimisation. Structural and Multidisciplinary Optimization 2008; 36(3):263-271.

51. Amir O, Sigmund O. Reinforcement layout design for concrete structures based on continuum damage and truss topology optimization. Structural and Multidisciplinary Optimization 2012; 47(2):157-174.

52. Amir O. A topology optimization procedure for reinforced concrete structures. Computers and Structures 2013; 114:46-58.

53. Jansen M, Lombaert G, Schevenels M, Sigmund O. Topology optimization of fail-safe structures using a simplified local damage model. Structural and Multidisciplinary Optimization 2013; 49(4):657-666.

54. James KA, Waisman H. Failure mitigation in optimal topology design using a coupled nonlinear continuum damage model. Computer Methods in Applied Mechanics and Engineering 2014; 268:614-631.

55. Kang Z, Liu P, Li M. Topology optimization considering fracture mechanics behaviors at specified locations. Structural and Multidisciplinary Optimization 2016; :1-18.

56. Verhoosel CV, de Borst R. A phase-field model for cohesive fracture. International Journal for Numerical Methods in Engineering 2013; 96(1):43-62.

57. Huang X, Xie YM. Bi-directional evolutionary topology optimization of continuum structures with one or multiple materials. Computational Mechanics 2009; 43(3):393-401.

58. Da DC, Cui XY, Long K, Li GY. Concurrent topological design of composite structures and the underlying multiphase materials. Computers and structures 2017; 179(1):1-14.

59. Maute K, Schwarz S, Ramm E. Adaptive topology optimization of elastoplastic structures. Structural Optimization 1998; 15(2):81-91.

60. Schwarz S, Maute K, Ramm E. Topology and shape optimization for elastoplastic structural response. Computer Methods in Applied Mechanics and Engineering 2001; 190(15-17):2135-2155.

61. Huang X, Xie YM. Topology optimization of nonlinear structures under displacement loading. Engineering Structures 2008; 30(7):2057-2068.

62. Xia L, Breitkopf P. Recent advances on topology optimization of multiscale nonlinear structures. Archives of Computational Methods in Engineering 2017; 24(2):227249.

63. Sigmund O. A 99 line topology optimization code written in matlab. Structural and Multidisciplinary Optimization 2001; 21(2):120-127.

64. Huang X, Xie YM. Topology Optimization of Continuum Structures: Methods and Applications. John Wiley \& Sons: Chichester, 2010.

65. Nguyen TT, Yvonnet J, Zhu QZ, Bornert M, Chateau C. A phase field method to simulate crack nucleation and propagation in strongly heterogeneous materials from direct imaging of their microstructure. Engineering Fracture Mechanics 2015; 139:18-39. 